\title{
Diacronie
}

Studi di Storia Contemporanea

$\mathrm{N}^{\circ} 32,4 \mid 2017$

Proiezioni individuali e agire collettivo nella storia

\section{Artisti popular fra contestazione e riflusso}

Il caso di Angelo Branduardi

\section{Carlo Bianchi}

\section{(2) OpenEdition \\ Journals}

\section{Edizione digitale}

URL: http://journals.openedition.org/diacronie/6566

DOI: $10.4000 /$ diacronie.6566

ISSN: 2038-0925

Editore

Association culturelle Diacronie

Notizia bibliografica digitale

Carlo Bianchi, « Artisti popular fra contestazione e riflusso », Diacronie [Online], № 32, 4 | 2017,

documento 4, Messo online il 29 décembre 2017, consultato il 01 mai 2019. URL : http://

journals.openedition.org/diacronie/6566; DOI : 10.4000/diacronie.6566 


\title{
Diacronie
}

Studi di Storia Contemporanea

$32,4 / 2017$

Proiezioni individuali e agire collettivo nella storia. Ruoli sociali, aspetti politici e nodi storiografici tra pubblico e privato

\section{Artisti popular fra contestazione e riflusso. Il caso di Angelo Branduardi}

\author{
Carlo BIANCHI
}

Per citare questo articolo:

BIANCHI, Carlo, «Artisti popular fra contestazione e riflusso. Il caso di Angelo Branduardi», Diacronie. Studi di Storia Contemporanea : Proiezioni individuali e agire collettivo nella storia. Ruoli sociali, aspetti politici e nodi storiografici tra pubblico e privato, 32, 4/2017, 29/12/2017,

URL: < http://www.studistorici.com/2017/12/29/bianchi_numero_32/ >

Diacronie Studi di Storia Contemporanea $\rightarrow$ http://www.diacronie.it

Rivista storica online. Uscita trimestrale.

redazione.diacronie@hotmail.it

Comitato di direzione: Naor Ben-Yehoyada - João Fábio Bertonha - Christopher Denis-Delacour - Maximiliano Fuentes Codera Anders Granås Kjøstvedt - John Paul Newman - Deborah Paci - Niccolò Pianciola - Spyridon Ploumidis - Wilko Graf Von Hardenberg

Comitato di redazione: Jacopo Bassi - Luca Bufarale - Gianluca Canè - Fausto Pietrancosta - Alessandro Salvador - Matteo Tomasoni - Luca Zuccolo

Diritti: gli articoli di Diacronie. Studi di Storia Contemporanea sono pubblicati sotto licenza Creative Commons 3.0. Possono essere riprodotti e modificati a patto di indicare eventuali modifiche dei contenuti, di riconoscere la paternità dell'opera e di condividerla allo stesso modo. La citazione di estratti è comunque sempre autorizzata, nei limiti previsti dalla legge. 


\title{
4/ Artisti popular fra contestazione e riflusso. Il caso di Angelo Branduardi
}

\author{
Carlo BIANCHI
}

L'Italia dei secondi anni Settanta, scossa dal terrorismo eppure sulla via del riflusso, vede imporsi nel panorama della popular music il cantautore Angelo Branduardi con testi di stampo favolistico avvolti da un misto di sonorità etniche e arcaiche che si innestano nel crepuscolo del progressive. Le teorie di Guy Debord e Pierre Bourdieu, soprattutto il rapporto "circolare" che Bourdieu individua fra artista e società, offrono elementi per una coerente spiegazione del fenomeno Branduardi, pilotato dal manager David Zard. Negli anni Ottanta dell'evasione definitiva, il cantautore si rivolge al mondo poetico di W. B. Yeats con un disco che sconfina nel campo di MUSOS e intellettuali. Ancora le teorie di Bourdieu aiutano a comprendere questo meccanismo e a contestualizzare alcune inedite dichiarazioni di Branduardi sulla sua traiettoria, che ha alternato adesioni e rifiuti, continuamente in bilico fra personale e collettivo.

\section{La musica del cambiamento}

Il calderone socio-culturale italiano degli anni Settanta aveva trovato un'espressione quantomai rispondente delle proprie istanze, derivate dal Sessantotto, nella musica popular etichettata come progressive. Il termine anglofono richiama lo stile sperimentale di alcuni complessi rock britannici comparsi con la "scena di Canterbury" negli anni Sessanta - formazioni che ricercavano nuovi linguaggi, sia in senso musicale sia testuale, tramite una commistione di generi diversi e fino ad allora indipendenti. Quel rock d'avanguardia faceva parte di un movimento giovanile più ampio, definibile "progressivo" tanto in senso musicale quanto sociale, che stava scuotendo molti altri paesi occidentali: nord-americani ed europei, perfino scandinavi, come la Svezia ${ }^{1}$, o socialisti, come l'Ungheria ${ }^{2}$. Anche nell'Italia figlia della contestazione, i

${ }^{1}$ Cfr. FORNÄS, Johan, il "Progetto Tenda" - Una manifestazione collettiva del movimento musicale progressivo giovanile degli anni '70, in FABBRI, Franco (a cura di), What is Popular Music? 41 saggi, interventi, ricerche sulla 
movimenti giovanili si identificavano con complessi rock che nel sonorizzare testi e convinzioni ideologiche aggiravano facili melodie prestabilite, inserivano le sezioni cantate in strutture formali superiori e mostravano, infine, un marcato carattere polistilistico.

Non è certo possibile trovare un'unica definizione per un panorama che anche solo in Italia era vario e nutrito tanto quanto le possibilità di sperimentazione musicale e le tematiche cui ispirare $\mathrm{i}$ testi ${ }^{3}$. Si passava da casi di esplicito schieramento politico nel sinistrismo come gli Area o gli Stormy Six divisi fra forti sperimentazioni etniche jazz-elettroniche (Area) e sonorità acustiche (Stormy Six) ad altri, come il Banco Del Mutuo Soccorso, che facevano dell'eclettismo musicale uno strumento di incontro fra le nuove diversità cresciute nel paese. Esisteva persino un progressive di ispirazione cattolica, sostanzialmente marginale, eppure sintomo di quanto le istanze di rinnovamento abitassero in tutto il tessuto sociale ${ }^{4}$. Invero, a prescindere dall'ambito di appartenenza, i nuovi climi sonori veicolavano raramente veri e propri messaggi politici. Più frequentemente evocavano invece mondi e modi di vita improntati a libertà di azione e pensiero, poetismi e aneliti alla creatività che allargavano gli orizzonti spirituali dell'uomo, al cospetto dell'«unica dimensione» stigmatizzata da Marcuse nella società industrializzata ${ }^{5}$. Il progressive era insomma un fattore fondamentale di quella «rivoluzione culturale» scaturita col Sessantotto che Arthur Marwick ha descritto in prospettiva internazionale ${ }^{6}$, e più di recente Gianmario Borio ha scandagliato in profondità con le sue competenze di filosofo e musicologo teorico ${ }^{7}$.

In Italia, la tendenza non coinvolgeva solo i complessi, ma anche cantanti e autori solisti - i quali, del resto, ricorrevano sempre al contributo di altri musicisti o parolieri per confezionare $\mathrm{i}$ loro prodotti. Alan Sorrenti, Franco Battiato, Juri Camisasca o Claudio Rocchi, avevano espresso ciascuno a suo modo le aspirazioni e i disagi della loro generazione sperimentando forme e sonorità inaudite nell'ambito del rock italiano. D'altronde, recenti studi hanno riconosciuto un carattere progressive anche ad alcuni cantautori che veicolavano contenuti sociali generalmente tramite le forme fisse della canzone strofica e ritornellata. Oltre a Fabrizio De André detto "il

musica di ogni giorno. II Conferenza Internazionale della IASPM. Reggio Emilia, IASPM e Musica/Realtà, 19-24 settembre 1983, Milano, Unicopli, 1983, pp. 261-266.

${ }^{2}$ SZEMERE, Anna, Il rock d'avanguardia in Ungheria, in FABBRI, Franco (a cura di), op. cit., pp. 285-289.

${ }^{3}$ Una catalogazione quantitativa utile soprattutto per la conoscenza di complessi poco noti è offerta da GABOLI, Alessandro, OTTONE, Giovanni, Progressive italiano, Milano, Giunti, 2007.

${ }^{4} \mathrm{Cfr}$. SALVARANI, Brunetto, SEMELLINI, Odoardo, Dio, tu e le rose. Il tema religioso nella musica pop italiana da Nilla Pizzi a Capossela, Trento, Il margine, 2013, pp. 36-41.

${ }^{5}$ MARCUSE, Herbert, L'uomo a una dimensione: l'ideologia della società industriale avanzata, Torino, Einaudi, 1967.

${ }^{6}$ MARWICK, Arthur, The Sixties. Cultural Revolution in Britain, France, Italy and the United States, c. 1958-1974, New York, Oxford University Press, 1998.

7 Oltre ai noti contributi accademici, va segnalata la preziosa sintesi pubblicata sul bimestrale «BresciaMusica» nel 2008 a seguito di una sua conferenza con Paolo Soddu al Museo S. Giulia di Brescia il 24 settembre di quell'anno, nell'ambito della manifestazione «Le dieci giornate di Brescia», durante la giornata dedicata al tema Il Sessantotto. A quarant'anni da una rivoluzione. Cfr. BORIO, Gianmario, «Una Rivoluzione culturale? Considerazioni sul Sessantotto», in BresciaMusica, 111, 10/2008, p. 15. 
progressivo" in un recente titolo di Franco Fabbri ${ }^{8}$, si considerino le complessità individuate da Alessandro Bratus negli album di Lucio Battisti Anima latina ${ }^{9}$ e Sì, viaggiare ${ }^{10}$. Nei circuiti musicali indipendenti dalle major discografiche e dalle relative politiche commerciali, nonché più attenti al patrimonio folklorico nazionale sulla spinta del Cantacronache e del Nuovo Canzoniere Italiano, spiccano alla fine del decennio alcuni progetti marcatamente politici di solisti in collaborazione, che condividono con il progressive, se non le matrici rock, i cross-over fra generi differenti da cui scaturiscono forme ampie e articolate, percorse altresì internamente da fili rossi musicali e narrativi. Nel 1978 Margot pubblicava il concept-album antinuclearista La messa dei villani nella cattedrale degli ingegneri in collaborazione con Virgilio Savona e il gruppo Strumento Concerto ${ }^{11}$, mentre Giovanna Marini intrecciava la propria voce e chitarra con polifonie popolari femminili (Correvano coi carri) $^{12}$ e col trio jazz Schiaffini-Iannaccone-Colombo (La Grande madre impazzita ${ }^{13}$ ).

\section{La canzone al centro}

Da un lato, l'aggettivo progressive emerge come un concetto-ombrello al quale si possono ricondurre gli ampliamenti e le innovazioni che caratterizzavano molte forme della popular music di quel periodo con le relative valenze antropologiche. D'altronde, era la forma della canzone, più delle musiche per l'intrattenimento (ritmi di danza, marcette e forme del beat) e più di altri generi altrettanto socializzanti (il blues) a costituire un principale raccordo in questo panorama eccezionalmente composito, per le sue caratteristiche di riconoscibilità, comprensibilità e riproducibilità. Modello culturale di facile acquisizione. Al cospetto di un calo di popolarità del Festival di Sanremo - si indeboliva un mezzo di diffusione dei valori legati all'egemonia cattolicodemocristiana $^{14}$ - i modelli della canzone venivano assorbiti da molti altri ambiti socio-culturali che corrispondevano a sorgenti di contenuti, forme e linguaggi assai connotati.

\footnotetext{
${ }^{8}$ FABBRI, Franco, De André il progressivo, in ID., Il suono in cui viviamo. Saggi sulla popular music, Milano, Il saggiatore, 2008, pp. 246-264. Ulteriori considerazioni in BIANCHI, Carlo, «Lo sguardo della contestazione. De André, Spoon River e il vento del Sessantotto», in Nuova Rivista Musicale Italiana, 3/2012, pp. 347-366.

${ }^{9}$ BATTISTI, Lucio, Anima latina, Numero Uno, 1974.

${ }^{10}$ ID., Amarsi un po'/Sì, viaggiare, Numero Uno, 1977. BRATUS, Alessandro, “"Anima latina" e "Sì, viaggiare" di Lucio Battisti e Mogol: una proposta analitica», in Rivista di Analisi e Teoria Musicale, XIII, 1/2007, pp. 103123.

${ }^{11}$ GALANTE GARRONE, Margot [Margherita], La Messa dei villani nella cattedrale degli ingegneri, Divergo, 1978.

${ }^{12}$ MARINI, Giovanna, Correvano coi carri. Cantata profana di Giovanna Marini, I dischi del sole, 1978.

${ }^{13}$ ID., La madre impazzita. Cantata e sonata, I dischi del sole 1979. Dal progetto è stato tratto anche un volume contenente la partitura: ID., La madre impazzita: Cantata e sonata di vita fantastica, mitica, inventata, Roma, R. Napoleone Editore, 1979.

14 «Il declino della manifestazione canora fu evidente fin dal 1973 (il "Festival della mutua", così lo definì il Corriere della sera) e sembrò irreversibile. Conseguenze immediate furono un drastico calo nella vendita dei dischi [...] un appannamento delle presenze [...] un ridimensionamento dell'appuntamento televisivo riservato alla sola serata finale». FACCI, Serena, SODDU, Paolo [con PILONI, Matteo], Il Festival di Sanremo. Parole e suoni raccontano la nazione, Roma, Carocci, 2011, pp. 185 et seq. Soddu e Facci notano che gli anni
} 
Qualche esempio. La canzone popular liturgica era entrata prepotentemente nelle celebrazioni e pratiche devozionali cattoliche, mentre quella folk sperimentata nei gruppi di ricerca popolare si trovava a misurarsi con la canzone politica di cantautori legata a gruppi extraparlamentari e movimenti sindacali di sinistra ${ }^{15}$. I cantautori mainstream venivano percepiti come "impegnati" o “alternativi" tramite meccanismi sovente controversi e interpretazioni arbitrarie delle loro canzoni ${ }^{16}$, accanto alle canzoni dei complessi rock inseriti nel sinistrismo i quali, oltre alle diversità di stile, si dividevano a loro volta in modo antagonista, eppure dialettico, fra produzioni discografiche major e indipendenti.

La canzone popular si poneva pertanto come luogo e mezzo privilegiato per convergenze e ibridazioni fra molteplici poetiche e significati. Tecnicamente, da un punto di vista musicale, essa incrociava generi afro-americani e repertori etnici, forme della musica sacra e barocca, fino alla composizione colta d'avanguardia. Tale fusione si faceva specchio ma anche fattore dell'incontroscontro fra tipologie umane e appartenenze sociali, aspirazioni al rinnovamento e al pluralismo che caratterizzavano tanto l'alta politica istituzionale del paese quanto i movimenti di contestazione "dal basso" trasversali alle logiche di partito e sostenitori di una politica intesa come partecipazione, più che come rappresentanza. Ideologie e comportamenti definibili genericamente di controcultura, che anche tramite la musica cioè sviluppavano una certa coscienza storica in opposizione a sistemi dominanti pregressi, trovavano altresì frequenti punti di contatto e scambi reciproci con la cultura dominante stessa nei suoi mutamenti di costume e mentalità - quantunque in Italia non si produsse mai un sovvertimento delle egemonie politiche.

\section{Collaborazioni Mainstream}

Riguardo all'ambito musicale popular di largo consumo, quello delle major discografiche con possibilità di larga distribuzione e promozione tramite mass-media, uno dei più eclatanti punti di incontro fra le tendenze progressive italiane e la figura di un cantautore si verifica nel 1978 con la produzione live internazionale nota come Carovana del Mediterraneo, ovvero l'incontro fra Angelo

Settanta costituiscono la fine del racconto unitario ed egemonico del paese e che la "rinascita" della manifestazione nel decennio successivo non potrà comunque competere con i fasti del ventennio precedente anche in ragione di una società italiana che era diventata «stabilmente plurale». Ibidem.

${ }^{15}$ Ad esempio Ivan Della Mea, Paolo Pietrangeli o Pino Masi. Per una panoramica accurata del fenomeno, fino ai nomi come Alfredo Bandelli e Piero Nissim, cfr. il volume del cantautore SANNUCCI, Corrado, Lotta continua. Gli uomini dopo, Arezzo, Limina, 1999.

${ }^{16}$ Oltre ai cantautori avvertiti come rappresentanti del sinistrismo politico per via di sporadici riferimenti nelle loro canzoni o dei loro atteggiamenti pubblici (ad es. Fabrizio De André, Francesco Guccini, Edoardo Bennato, Francesco de Gregori) è sintomatico di tali oscillazioni ermeneutiche il caso di Lucio Battisti, perché proprio il carattere intimista e a-politico dei testi, scritti da Giulio Rapetti (Mogol), ne ha consentito l'identificazione con i movimenti di destra. Cfr. DI GIORGI, Cristina, FERRARIO, Ippolito E., Il nostro canto libero. Il neofascismo e la musica alternativa: lotta politica e conflitto generazionale negli anni di piombo, Roma, Castelvecchi, 2010. 
Branduardi, giunto all'apice del successo, e il Banco del Mutuo Soccorso, uno dei complessi progressive italiani dallo spettro stilistico più ampio, ma si aggregavano anche i gruppi etnici di due musicisti collaboratori dei dischi di Branduardi: il violinista Felix Mizrahi, proveniente dalla comunità ebraica d'Egitto, e il suonatore di launeddas Luigi Lai, con il Gruppo sardo S. Gemiliano di Sestu. L'attenzione verso la musica etnica era una delle nuove istanze veicolate dal progressive italiano $^{17}$. Nondimeno, in generale, emergeva da questo quadro di collaborazioni una poetica progressive in quanto i complessi più rappresentativi di quella tendenza erano spesso formati da musicisti che provenivano da ambienti sociali diversi, ognuno col rispettivo percorso formativo. Cosa che contribuiva a rendere il progressive un precipuo prodotto di sintesi: musicale, ma anche culturale.

Ai tempi della Carovana del Mediterraneo, la formazione del BMS era costituita dal cantante Francesco di Giacomo, dai fratelli Vittorio e Gianni Nocenzi, entrambi pianisti-tastieristi (il secondo anche clarinettista), dal bassista Gianni Colaiacomo, dal chitarrista e cornista Rodolfo Maltese e dal batterista Pierluigi Calderoni. Come ha ricordato di recente Vittorio Nocenzi, l'iniziativa era scaturita da David Zard, manager comune di Branduardi e del BMS ${ }^{18}$. Tuttavia, già in precedenza Branduardi aveva messo violino e competenze da poliglotta al servizio dell'album Come in un'ultima cena del $\mathrm{BMS}^{19}$, tradotto in inglese come As in a Last Supper, mentre Gianni Nocenzi aveva partecipato alla registrazione dell'album Alla fiera dell'Est. E le analogie e la comunanza di passioni andavano ben oltre. Era la predilezione per lo strumentismo unita a quella per la ricerca etnica il fattore che rese poi naturale anche la collaborazione dal vivo fra il BMS e Branduardi unita ai gruppi etnici. Le intersezioni fra il BMS e la musica etnica sono emblematiche nelle collaborazioni di Vittorio Nocenzi con Luigi Lai e Luigi Cinque ${ }^{20}$, e nei suoi studi di etnomusicologia con Diego Carpitella all'università di Roma ${ }^{21}$. La successiva collaborazione con Branduardi nella Carovana del Mediterraneo avrebbe spinto Nocenzi a scrivere il Canto di primavera ${ }^{22}$, fusione di un canto secentesco dell'Alta Savoia e uno palestinese, veicolando gli ideali di solidarietà della band, ma i punti di contatto con lo stile di Branduardi risiedevano anche nelle matrici classiche. L'idea della Carovana infatti giungeva subito dopo l'album del BMS ...di terra registrato con l'orchestra sinfonica del Conservatorio "S. Cecilia" di Roma (diretta da Antonio Scarlato) che rappresentava il più ambizioso sconfinamento della formazione romana nello stile

\footnotetext{
${ }^{17}$ In aggiunta alle considerazioni che seguono sull'attività di Vittorio Nocenzi e del BMS, cfr. la testimonianza di Mauro Pagani in BIANCHI, Carlo «Dal "progressive” alla canzone d'autore. Lungo i sentieri di Mauro Pagani», in Musica/Realtà, 92, 2/2010, pp. 115-140, pp. 135-137.

${ }^{18}$ Intervista con l'autore, "Quella strana magia degli anni Settanta. Vittorio Nocenzi a Brescia con il "Banco"», in BresciaMusica, 153, 2/2017, pp. 10-11.

${ }^{19}$ BANCO DEL MUTUO SOCCORSO, Come in un'ultima cena, Manticore, 1976.

${ }^{20}$ Cfr. SALVATORE, Gianfranco, Vittorio Nocenzi. Sguardi dall'estremo occidente, Viterbo, Stampa alternativa, 2011, pp. 81-87.

${ }^{21}$ Ibidem, pp. 13 et seq.

${ }^{22}$ BANCO DEL MUTUO SOCCORSO, Canto di primavera, in ID., Canto di primavera, Ricordi, 1979.
} 
strumentale classico - uno dei progetti cui lo stesso Nocenzi afferma di sentirsi legato di più ancora oggi ${ }^{23}$.

Da parte sua Branduardi, prima della svolta etnica che lo avrebbe reso un fenomeno di massa, aveva inciso due album per la RCA rispettivamente nel 1974 e nel 1975 che denotavano una chiara matrice progressive. Questa derivava in parte dalla formazione violinistica classica di Branduardi, al "Paganini" di Genova, che egli aveva accostato fin dall'adolescenza alla scrittura di canzoni con la chitarra. Ancor più decisivo era stato l'incontro con l'arrangiatore e compositore britannico Paul Buckmaster: musicista passato attraverso esperienze diversissime, già violoncellista-bassista della Third Ear Band e direttore del Chitinous Ensemble, aveva collaborato come arrangiatore a dischi di Miles Davis, Elton John e Rolling Stones. Il contatto avvenne su iniziativa di Branduardi, dopo che la RCA aveva rigettato i materiali di un suo primo disco. «A quel tempo Buckmaster era un personaggio assolutamente sulla cresta dell'onda» - ricorda il cantautore. «Gli scrissi una lettera, che immaginavo sarebbe stata cestinata, a cui avevo allegato una cassetta, che lui però non ascoltò mai. Semplicemente, da uomo d'estro qual era, ritenne che quello che avevo scritto nella lettera valesse l'ipotesi di una collaborazione. Io gli devo moltissimo, per tante cose, per tutto quello che ho imparato sul trattamento dei suoni.... ${ }^{24}$.

Buckmaster produce Angelo Branduardi '74 orchestrando archi e fiati, aggiungendo alle canzoni accompagnamenti, sottofondi e intermezzi recitati nella direzione del concept-album richiesto dai discografici (altro contenitore tipicamente progressive). Così, l'esordio di Branduardi, voce, chitarra, flauti e violino in mano, avviene nel segno di una canzone ispiratagli sì da alcuni modelli cantautorali (Donovan, Dylan e Brassens, e soprattutto Cat Stevens), ma altresì contaminata con suoni e linguaggi moderni a tratti anche complessi. Nel disco successivo, La luna, pur senza Buckmaster, l'impronta progressive permane, come notano anche Riccardo Storti e Fabio Zuffanti $^{25}$, grazie all'apporto di alcuni musicisti fra cui Maurizio Fabrizio, arrangiatore e anche lui polistrumentista formatosi al conservatorio di Milano, da cui Branduardi non si separerà più. Oltre alla creatività musicale, La luna conferma anche i temi poetici e le narrazioni senza tempo del primo disco - «la linea così tenue tracciata fra realtà e fantasia» nei versi che il cantautore scrive per una delle sue poche canzoni d'amore - nonché la vena trobadorica che si profilava fin dall'inizio ricorrendo a melodie popolari. Sono infine un marchio gli accompagnamenti chitarristici talvolta raffinati. Spiccano i fraseggi di Confessioni di un malandrino, poesia di Sergej

\footnotetext{
${ }^{23}$ BANCO DEL MUTUO SOCCORSO, ...di terra, Ricordi, 1978. Cfr. BIANCHI, Carlo, Quella strana magia degli anni Settanta, cit., p. 11.

${ }^{24}$ Intervista con il canale tv italiano Videomusic, 1990, servizio relativo al disco Il ladro.

${ }^{25}$ STORTI, Riccardo, ZUFFANTI, Fabio, Prog Rock. 101 dischi dal 1967 al 1980, Roma, Arcana, 2017, pp. 247-250.
} 
Esenin nella traduzione di Renato Poggioli, con le chitarre classiche di Branduardi e Fabrizio in un intreccio che attirerà l'interesse di riviste specializzate ${ }^{26}$.

\section{4. "Qui e ora" nonostante tutto}

A questo punto avviene la svolta, ma non quella preconizzata da certi discografici. Come Branduardi ha rimarcato in varie circostanze, erano forti le pressioni per scrivere canzoni che parlassero di ciò che accadeva nel paese alla metà degli anni Settanta, fra contestazioni, rivendicazioni operaie e strategie della tensione, incombenti gli anni di piombo. L'attenzione alla cronaca sarebbe un modo per vendere di più. Invece, un altro incontro, quello con David Zard e suo fratello fratello Dory, accentua le propensioni favolistiche di Branduardi, unite a quelle della moglie Luisa Zappa, già sua collaboratrice per i testi come Maurizio Fabrizio lo è per la musica, sospingendo la fantasia verso zone ancora più lontane. Alla fiera dell'est, album inciso ora per la Polydor, presenta come title track un antico canto della Pasqua ebraica suggerito dallo stesso Zard, pure lui giunto in Italia come profugo fra gli ebrei di Libia. Nel disco ci sono altri brani folk dell'Europa periferica, fra cui già si affacciano con forza quelli celtici (scozzesi o irlandesi) ricercati alle fonti nelle Child Ballads oppure in gruppi come i Pentangle o ancora nelle collaborazioni con Alan Stivell.

Nonostante Branduardi col suo entourage fosse stato messo in guardia da certi produttori - «se lei non parla di piazze, fabbriche e cortei, non andrà da nessuna parte. Altro che il topolino, il gatto e l'angelo della morte ${ }^{27}$ - Alla fiera dell'est resiste a un'iniziale indifferenza del mercato e finisce col riscuotere un poderoso successo di vendite; ma vince anche il premio della critica discografica italiana 1977. «Non lo dico con tono polemico, ma il direttore artistico della Polydor, non era italiano» - avrebbe rimarcato il cantautore molto tempo dopo ${ }^{28}$. I riconoscimenti artistici e commerciali proseguiranno ancora più soddisfacenti, anche all'estero, con i due album successivi, La pulce d'acqua (1977) e Cogli la prima mela (1979, inciso in Germania). La formula risulta vincente sia per motivi musicali, sia testuali. L'apertura di un contenitore di massa come la canzone popular verso la musica etnica andava di pari passo con un'ansia di rinnovamento sociale che da tempo si profilava in quanto apertura alle culture lontane e agli strati subalterni delle popolazioni, ma era in generale la fantasia e l'immaginazione che le istanze del Sessantotto avrebbero voluto mandare al potere, l'altra matrice profonda.

\footnotetext{
${ }^{26}$ Cfr. nota 59.

${ }^{27}$ Frase raccolta dall'autore in più di una circostanza. Cfr. anche l'intervista con BERNIERI, Claudio, Non sparate sul cantautore. Canzoni come pietre, musica sotto tiro, Milano, Mazzotta, 1978, pp. 222-223.

${ }^{28}$ Frase raccolta dall'autore nel 1997.
} 
Vi erano vari legami, al cospetto delle differenze, fra questi dischi e la precedente produzione di Branduardi per la RCA. Da un lato, permaneva una tendenza all'ampliamento strumentale. D'altronde, anche le sonorità etniche non erano una novità improvvisa nella produzione del cantautore e nel suo orizzonte. Come abbiamo accennato in apertura, l'attenzione verso elementi etnici era parte, a sua volta, di quel retroterra culturale da cui era scaturito il progressive, e non si poneva solo come veicolo di elaborazione musicale, ma in quanto istanza generale della controcultura del Sessantotto. Già in precedenza, la riscoperta e la valorizzazione di valori etnici, sia autoctoni sia di paesi lontani, aveva fatto parte di diversificate tendenze musicali popular. Gli studi di etnomusicologi come Roberto Leydi e Diego Carpitella, Ernesto De Martino o Roberto De Simone avevano informato direttamente gruppi e movimenti di folk revival come Nuovo Canzoniere Italiano, nato sulle ceneri del Cantacronache, o il Canzoniere del Lazio e la Nuova Compagnia di Canto Popolare, ma negli anni Settanta lo spirito di accoglienza verso ambiti popolari o etnie diverse si ripercuoteva all'interno di vari generi musicali popular e a vari livelli, da quelli più lontani dalle culture dominanti fino alle più commerciali logiche di consumo, e anche tramite processi assai differenziati.

Moni Ovadia, discepolo di Leydi, col gruppo folk internazionale poteva incidere sul percorso di un solista come Mauro Pagani - già attratto dalle culture "altre" a dispetto del rock occidentale della PFM. Il Canzoniere del Lazio poteva trovare collaborazione in Demetrio Stratos - già proiettato verso la ricerca etnica esotica, sia come solista, sia come membro degli Area. Roberto de Simone oltrepassava la Nuova Compagnia di Canto Popolare al tempo di Musicanova e creava un'opera di contaminazione classico-popolare con La gatta cenerentola - ma arrangiava persino alcuni dischi di Edoardo Bennato. Certe uscite di Vittorio Nocenzi col BMS potevano attingere indifferentemente a Bartók come agli studi di Carpitella che abbiamo ricordato. Si era formata insomma una fitta rete di scambi che trovavano un denominatore comune nelle ideologie del sinistrismo, e che non dipendeva solo dalle collaborazioni personali, ma anche dalla maggiore circolazione dei dischi, sia di musica folk italiana (si pensi ai Dischi del sole) sia provenienti dall'estero.

In linea con la "alterità" delle etnie esotiche, anche il côté favolistico e a-temporale dei testi costituiva un fattore di continuità portato all'estremo, rispetto ai movimenti precedenti, sia nel personale percorso discografico di Branduardi, sia in una prospettiva collettiva. Per quanto la contestazione anti-establishment che percorreva gli anni Settanta potesse avere obiettivi assai concreti, le espressioni culturali giovanili si appuntavano spesso su narrazioni fiabesche, evocazioni di mondi lontani e invocazioni alla fantasia come modello per le scelte di vita e comportamentali. L'intreccio con la contestazione politica produceva una sovrapposizione di favola e concretezza. Basti pensare che uno dei movimenti extraparlamentari più seguiti anche da un punto di vista musicale, Re Nudo, originariamente doveva addirittura chiamarsi Cappuccetto 
Rosso per via della fiaba che si tingeva di rosso inteso come colore comunista ${ }^{29}$. I testi di certi complessi politicamente schierati, come gli Area, potevano avere anche riferimenti espliciti ad accadimenti e ideologie, eppure la creatività che allargava il mondo del reale e del concreto, spesso con l'effetto voluto di spiazzare l'ascoltatore, si annidava perfino in quelle canzoni.

Le dimensioni del fenomeno Branduardi veicolato dalla Carovana del Mediterraneo e dalle produzioni live successive hanno stimolato vari tentativi di interpretazione sociologica, alla ricerca di modelli di identificazione di massa. Il più accurato finora pare quello di Enrica Tedeschi, che ravvisa appunto negli anni Settanta una generale tendenza a modalità di comunicazione delle tensioni e dei confitti presenti tramite linguaggi indiretti ${ }^{30}$. Pare sfuggire tuttavia alla sociologa romana la relazione altrettanto coerente con lo spirito del riflusso alla fine del decennio, quando le istanze della contestazione si stanno esaurendo. L'identificazione con la fase del riflusso era stata già stata notata a quel tempo, e stigmatizzata, da due critici come Giampiero Comolli ed Enzo Gentile che intravvedevano nel fenomeno Branduardi un emblematico affievolirsi dell'impegno sociale dei cantautori ${ }^{31}$. Anche Comolli intravede il controverso legame con i precedenti linguaggi del corpo e le pratiche di condivisione del piacere e dell'immaginazione, ma non riesce a sciogliere la contraddizione rispetto alle caratteristiche del riflusso, come non vi riesce Enrica Tedeschi nel sottoporre a critica la spiegazione di Comolli ${ }^{32}$.

\footnotetext{
${ }^{29}$ Per considerazioni sull'ideologia del movimento, cfr. VALCARENGHI, Andrea, Underground: a Pugno Chiuso!, Roma, Arcana, 1973.

${ }^{30}$ TEDESCHI, Enrica, Colori. L'immaginazione musicale di Angelo Branduardi, Roma, Philos, 1998, pp. 29-30.

${ }^{31}$ COMOLLI, Giampiero, Angelo Branduardi. Canzoni, Roma, Lato Side, 1979; GENTILE, Enzo, Guida critica ai cantautori italiani, Milano, Gammalibri, 1979, pp. 25-27. Feroce in particolare la critica di Gentile. Data una sua visione che premia, nostalgicamente, poetiche e movimenti giovanili politicizzati in declino, e svaluta i cantautori compromessi con la grande industria discografica (il solo Edoardo Bennato riscuote un giudizio del tutto positivo), Gentile afferma che Branduardi «appartiene di diritto a quella categoria di musicisti restaurati, riverniciati a nuovo da un impresario furbastro e portati come in un bel sogno al successo internazionale. Capita anche questo nella musica leggera in Italia, dove $\mathrm{i}$ talenti riescono ad essere emarginati, mentre coloro che sanno mistificare e contrabbandare sé stessi con un pizzico di classe e di savoir faire, quasi inevitabilmente sfondano». Ibidem, p. 25.

${ }^{32}$ TEDESCHI, Enrica, Colori, cit., p. 32.
} 


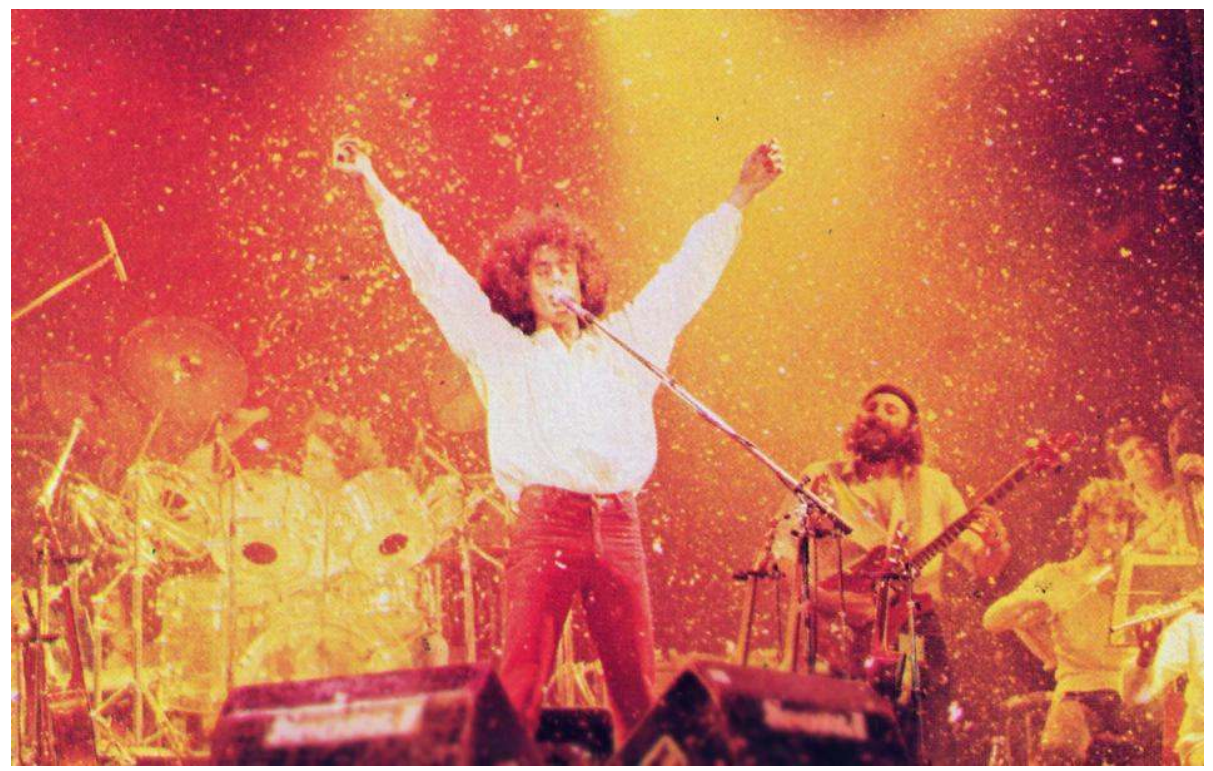

Figura 1. Tournée (1979), produzione Zard.

Il problema è che se la formula-Branduardi, pure accusata di evadere la realtà, risultava vincente proprio per questo, era perché essa si faceva tanto specchio quanto fattore di un processo sociale altrettanto antitetico e controverso. Quando l'operazione condotta da David Zard raggiunge i massimi risultati in termini di vendite e di affluenza ai concerti, contribuisce a riversare nel circuito e nel mercato giovanile di largo consumo i germi di una controcultura che nel generarsi aveva posto le basi per il proprio superamento. Tanto cioè l'immaginazione e la fantasia avevano avuto un ruolo seminale nella contestazione di un establishment ritenuto asfittico, quanto esse iniziavano a rivelarsi un veicolo di disimpegno da quelle stesse istanze rivoluzionarie e liberatorie. Così, finivano col sovrapporsi tendenze in entrambi i sensi. Nell'ambito della musica giovanile, si pensi alla comparsa della disco-music, emblema di una generazione che inizia a lanciarsi nel divertimento e nell'edonismo, e contemporaneamente al movimento Rock in opposition o all'attività della Cooperativa L'orchestra ${ }^{33}$. E molto del rock progressive che permane inizia a cedere in quantità e carica inventiva.

La carovana del Mediterraneo creata da Zard contribuiva a incrementare l'impatto mediatico della figura di Branduardi recando la sua idea di Concerto come "festa", invece che come raduno in nome di ideali, confronti e dibattiti anche accesi, trovando altresì un fulcro in quelle suggestioni "mediterranee" che per altri versi coinvolgevano autori italiani reduci da un passato progressive (Mauro Pagani) ${ }^{34}$, o addirittura politico (Pino Masi) ${ }^{35}$. Branduardi partecipava alla

\footnotetext{
${ }^{33}$ FABBRI, Franco, «Orchestral Manoeuvres in the 1970s: l'Orchestra Cooperative, 1974-1983», in Popular Music, 26, 3/2007, pp. 409-427.

${ }^{34}$ MAURO PAGANI, Mauro Pagani, Ascolto, 1978 è il primo album etnico di Pagani dopo il suo distacco dalla PFM avvenuto nel 1976. Cfr. BIANCHI, Carlo, Dal "progressive" alla canzone d'autore. Lungo i sentieri di Mauro Pagani, cit., p. 136.
} 
musica e alle canzoni del BMS con violino e voce, e allo stesso tempo le sue storie senza tempo né luogo risultavano musicalmente più articolate rispetto alle versioni originali, grazie all'apporto del BMS come dei gruppi entici di Felix Mizrahi e Luigi Lai. Le caratteristiche della Carovana del Mediterraneo col suo impatto pubblico sono documentate in parte dal triplo disco live Concerto, edito nel 1980 [fig. 1-5], che fissa alcuni brani di un concerto all'Arena di Verona del 15 settembre 1978, insieme alle registrazioni di altre date successive alla Carovana del Mediterraneo, effettuate nel 1979 in Francia, Germania, Svizzera e Inghilterra dopo l'uscita dell'album Cogli la prima mela, sempre con la produzione dei fratelli Zard e con caratteristiche tecniche molto simili - fra le varie cose, l'aggiunta di una sezione di $\operatorname{archi}^{36}$.

\section{Figura 2. Carovana del mediterraneo}

(1978): Luigi Lai alle launeddas.

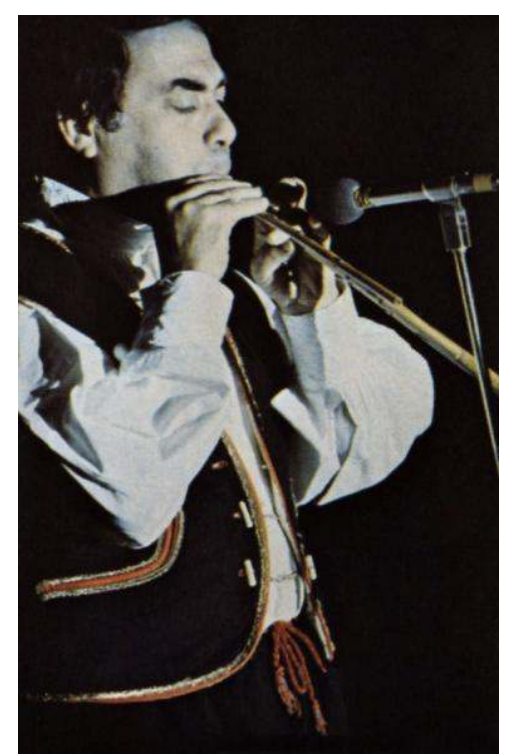

L'intreccio di tendenze nelle produzioni live di Zard indicava come anche il progressive, in quanto espressione di una seminale contro-cultura giovanile, avesse recato in sé i germi del proprio superamento artistico e ideologico tramite la ricerca del diverso e di altre dimensioni. Ovviamente il passaggio da un estremo al suo opposto avveniva gradualmente, oscillando, in modo ambiguo e talvolta contraddittorio, quando non conflittuale. Proprio il BMS pur avendo commentato a modo proprio il colpo di stato in Cile (Io sono nato libero) aveva dapprima trovato metafore sociali non immediate riandando all'età della pietra (Darwin! $\left.{ }^{37}\right)$. Riguardo ai cantautori mainstream, i concept-album di Edoardo Bennato (Burattino senza fili; Sono solo Canzonette ${ }^{38}$ ) o Alberto Camerini (Cenerentola e il pane quotidiano; Gelato metropolitano ${ }^{39}$ ) offrivano narrazioni favolistiche dove certi elementi di critica socio-politica potevano essere incistati con maggior efficacia tramite quelle immagini tenui, ma allo stesso tempo, a seconda dei punti di vista, risultare stemperati dalle medesime tematiche universali innescando fatalmente nell'audience effetti controversi. Era ancora emblematico che nel 1978 proprio la partecipazione di Branduardi alla leggenda di Samarcanda, narrata da Vecchioni sul

\footnotetext{
${ }^{35}$ MASI, Pino, Alla ricerca della madre mediterranea, Cramps, 1977.

${ }^{36}$ Viene spesso fatta confusione, anche su voci online come Wikipedia, fra la Carovana del mediterraneo e le tournée successive. La prima risale al 1978, quelle successive sono altre produzioni, seppure con caratteristiche simili. Infatti, sui dischi Polydor del cofanetto Concerto viene indicata la data del 15/9/1978 come facente parte della Carovana del mediterraneo, le altre invece no.

${ }^{37}$ BANCO DEL MUTUO SOCCORSO, Io sono nato libero, Ricordi, 1973; ID., Darwin!, Ricordi 1972.

${ }^{38}$ BENNATO, Edoardo, Burattino senza fili, Ricordi, 1977; ID., Sono solo canzonette, Ricordi, 1980.

${ }^{39}$ CAMERINI, Alberto, Cenerentola e il pane quotidiano, Cramps, 1976; ID., Gelato metropolitano, Cramps, 1977.
} 
disco omonimo, venisse seguita dallo "sparo di pistola" della canzone Vaudeville che celebrava ironicamente l'attacco politico a un De Gregori ritenuto dai contestatori troppo immaginativo (o intimista) e poco engagé $e^{40}$.

La carica festante della Carovana del Mediterraneo e delle produzioni successive si sovrapponeva all'atmosfera di scontro ai concerti talvolta al limite della guerriglia che il terrorismo e le strategie della tensione avevano contribuito a creare. Lo stesso Branduardi ne era stato già coinvolto - quando aveva esordito come spalla di Lou Reed in un concerto romano del 1975 caratterizzato da violenti tafferugli ${ }^{41}-\mathrm{ma}$ anche in seguito ${ }^{42}$. Da parte sua, egli ha sempre asserito di non voler comunicare messaggi precisi tramite le sue canzoni. «Il mio modo di scrivere non è "qui ed ora", ma "altro ed altrove"» avrebbe dichiarato spesso, salvo poi aggiungere «anche se poi è tutto da vedere, perché uno scrive in un certo modo perché comunque respira un certo tipo di aria. Se vivesse in un altro momento e in un altro luogo, scriverebbe in un altro modo» ${ }^{43}$. Né avrebbe misconosciuto la propria appartenenza a una generazione cresciuta sulle istanze del Sessantotto $^{44}$.

40 L'episodio rievocato è quello del concerto al Palalido di Milano nel 1976 su cui si pronunciò pubblicamente lo stesso De Gregori. Cfr. DEREGIBUS, Enrico, Quello che non so, lo so cantare. Storia di Francesco De Gregori, Giunti, Firenze 2003, pp. 93-94; FABRETTI, Claudio, Francesco De Gregori. Fra le pagine chiare e le pagine scure, Roma, Arcana, 2011, pp. 88-89.

${ }^{41}$ Cfr. la testimonianza di Vittorio Nocenzi in, BIANCHI, Carlo, Quella strana magia degli anni Settanta, cit., pp. 10-11; BIACCHESSI, Daniele, Storie di Rock Italiano. Dal boom economico alla crisi internazionale, Milano, Jaca Book, 2016, pp. 102-103.

${ }^{42}$ BERNIERI, Claudio, Non sparate sul cantautore, cit., p. 221.

${ }^{43}$ Intervista televisiva sul canale italiano Videomusic del febbraio 1993, Special relativo al disco Si può fare (EMI 1992) URL: < https://www.youtube.com/watch?v=jcT_axL-ehg > [consultato il 12 novembre 2017].

${ }^{44}$ Intervista trasmessa da Radio Radicale il 20/4/1984 a Roma in occasione del Concerto contro la fame per tre milioni di vivi subito, URL: < https://www.youtube.com/watch?v=0JsXnVo-TbQ > [consultato il 12 novembre 2017]. 


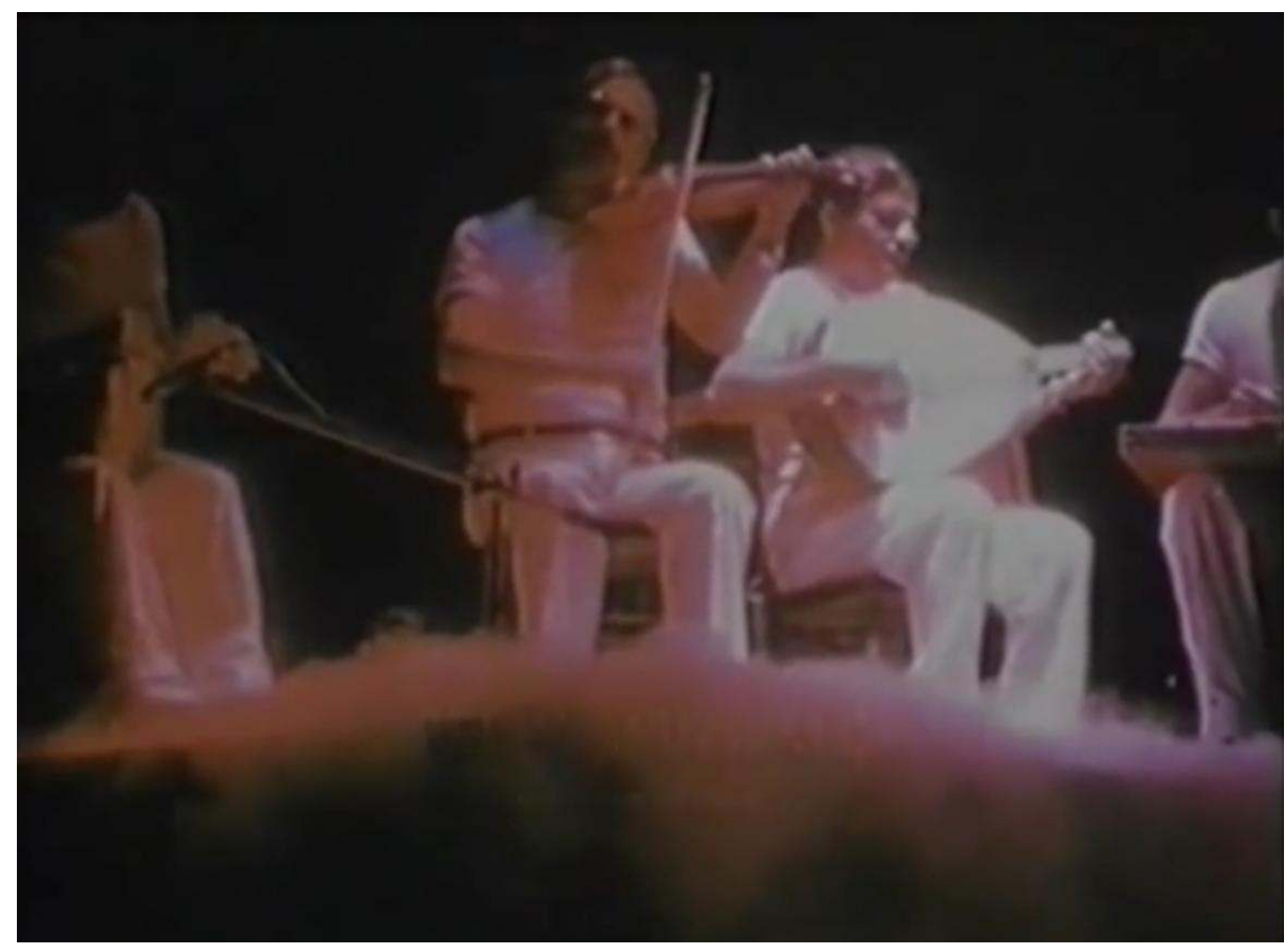

Figura 3: Carovana del mediterraneo (1978): Felix Mizrahi e il suo gruppo

Nei dischi incisi per la Polydor, la predilezione per canti e sonorità etniche si accompagna a quella per musiche antiche. Pure questa era una tendenza che caratterizzava altre ricerche di ambito musicale popular in quel periodo, derivata dalle coincidenze fra moduli musicali esotici e arcaici, e che in senso culturale generale può essere vista sempre nell'ottica dell'“altro e altrove", ora nello spazio, ora nel tempo - considerando inoltre che anche le melodie popolari risalgono in genere a tempi remoti. D'altronde, i moduli etnico-arcaici venivano re-impiegati da Branduardi e dai suoi collaboratori per creare uno stile frammisto in cui a fianco dei prestiti, dichiarati o taciti, spiccano anche canzoni del tutto originali e in cui pure certe citazioni eclatanti si trovano ristrumentate e ri-arrangiate anche da un punto di vista formale. Ad esempio, la Schiarazula marazula, dal primo Libro di balli di Mainerio è preceduta da una lunga introduzione strumentale, marcata dalle launeddas di Luigi Lai, e in seguito aggiunge sezioni di raccordo per un'alternanza strofa-ritornello che conferisce maggiore impatto al ritorno della melodia iniziale. La celebre melodia popolare Henry Martin dalle Child Ballads scozzesi, impiegata per Il funerale (su una poesia rielaborata di Franco Fortini) viene introdotta anch'essa da un'ampia elaborazione strumentale che mischia elementi etnici con espedienti timbrici e rumoristici tipici invece della musica colta d'avanguardia. 
Al centro di tutto però rimane sempre la forma della canzone, fosse originale o frutto di riarrangiamenti e libere ispirazioni. Una delle chiavi del successo era l'impiego di moduli di scrittura «semplici ma allo stesso tempo inconsueti» come ha dichiarato Branduardi a proposito delle Child Ballads ${ }^{45}$, ma il principio può essere esteso ad altre forme e fonti di ispirazione, che altrettanto potevano avere un effetto dirompente pur nella loro semplicità di base. D'altronde gli arrangiamenti eclettici ed estesi, talvolta complessi, avevano l'effetto di renderle recepibili in un ampio contesto popular moderno. Un altro esempio indicativo è costituito dalla canzone Alla fiera dell'est. L'espansione continua della strofa consente un progressivo ispessimento della dinamica e del tessuto orchestrale e una continua aggiunta di contro-melodie, affidate a strumenti classici o popolari, mentre gli intermezzi riportano il violino di Branduardi alla matrice mediorientale dello strumento. Senza però che tutto questo vada a scapito della facilità di ascolto, grazie alla ripetizione della cantilena, alle semplici armonie e all'aggiunta della sezione ritmica bassobatteria.

Al carattere inconsueto di queste forme, anche nelle tipologie più semplici, concorreva poi l'impiego di strumenti etnici rari per il repertorio cantautorale italiano mainstream. A fianco di organici pop e orchestrazioni classiche, nei dischi prodotti per la Poydor si odono ocarine, arpe celtiche, sitar, bouzouki, flauti di pan, launeddas, cetre e strumenti della tradizione araba che suonano quarti di tono. Al di là di un'identificazione con tendenze della società di massa, il successo di questa produzione derivava anche da oggettive qualità tecniche come appunto la cura nella ricerca organologica, la perizia negli arrangiamenti, e l'apporto di ottimi musicisti sia in studio sia dal vivo. Last but not least, era determinante la figura poliedrica dello stesso Branduardi: a metà fra musicista e cantautore, di voce peculiarmente musicale, già da adolescente appassionato di letteratura in musica, addirittura studente di Franco Fortini fra i banchi di un liceo per il turismo, nonché, infine, personalità di spicco che si valorizzava appieno nel ruolo di carismatico performer dal vivo. In gergo: animale da palco. E con questo giungiamo a un altro aspetto fondamentale di tutto il discorso.

\footnotetext{
${ }^{45}$ «A quel tempo per me era importante tutta la musica popolare ricavata dal periodo romantico... James Childs era uno di quelli che in epoca romantica, quando andava di moda recuperare le tradizioni, si era messo a ricercare materiale popolare prodotto nelle epoche precedenti. In queste ballate ci sono tutta una serie di precisi modi di scrivere, peraltro molto semplici ma assolutamente inconsueti, con testi che si riferiscono a storie, a fiabe nordiche, e le parole spesso sono molto violente» (BIANCHI, Carlo, «Il musicista non è un cantante. Intervista ad Angelo Branduardi. In occasione di un recente "Happening"", in BresciaMusica, 55, 1/1997, p. 20).
} 


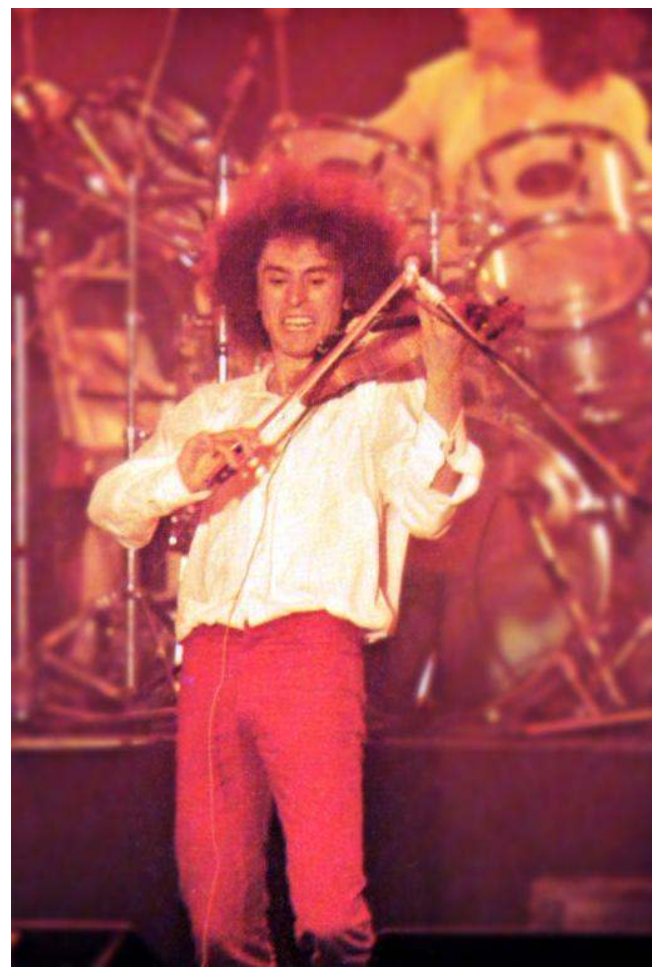

Figura 4: Tournée (1979), produzione Zard.

\section{Società dello spettacolo}

Gli ingredienti che David Zard aveva rilevato per questa produzione non erano solo intrinsecamente musicali e testuali, ma appunto performativi e di immagine. Il successo di quelle tournée risiedeva anche in una dimensione altamente spettacolare: allestimenti per quel tempo tecnologicamente avanzati ${ }^{46}$, imponenti impianti di amplificazione con intere pareti di casse, danzatori sul palco, luci colorate nei cieli notturni ed effetti speciali, su cui il front-man Branduardi si stagliava peculiare anche in quanto figura fisica, nella sua magrezza longilinea sormontata da un cespuglio di capelli. Immagini e suoni sul palco che catturavano l'attenzione del maggiore mass-medium, la televisione (Fig. 6), ma le pratiche performative attivate e catalizzate erano anche quelle del pubblico, sia quelle "implicite" sia "esplicite" - seguendo la distinzione di Enrica Tedeschi. Le prime implicite in quanto «forme di auto-rappresentazione indiretta come la proiezione sul personaggio-cult di modelli di comportamento e valori simbolici che rivestono particolare significatività per l'audience ${ }^{47}$, le seconde esplicite in quanto performance vere e proprie, con gli spettatori coinvolti non solo nel canto ma addirittura fisicamente nelle danze, grandi girotondi di giovani mano nella mano.

\footnotetext{
${ }^{46}$ Perfino Enzo Gentile, nella sua ideologica avversione alla formula Branduardi, rimarcava questo aspetto come distintivo rispetto ad altri cantautori - «Branduardi è seguito in concerto da apparecchiature mega e da squadre di tecnici che approntano uno spettacolo molto pulito e preciso». GENTILE, Enzo, Guida critica ai cantautori italiani, cit., p. 27.

${ }^{47}$ TEDESCHI, Enrica, Vita da Fan, Roma, Meltemi, 2003, p. 98.
} 


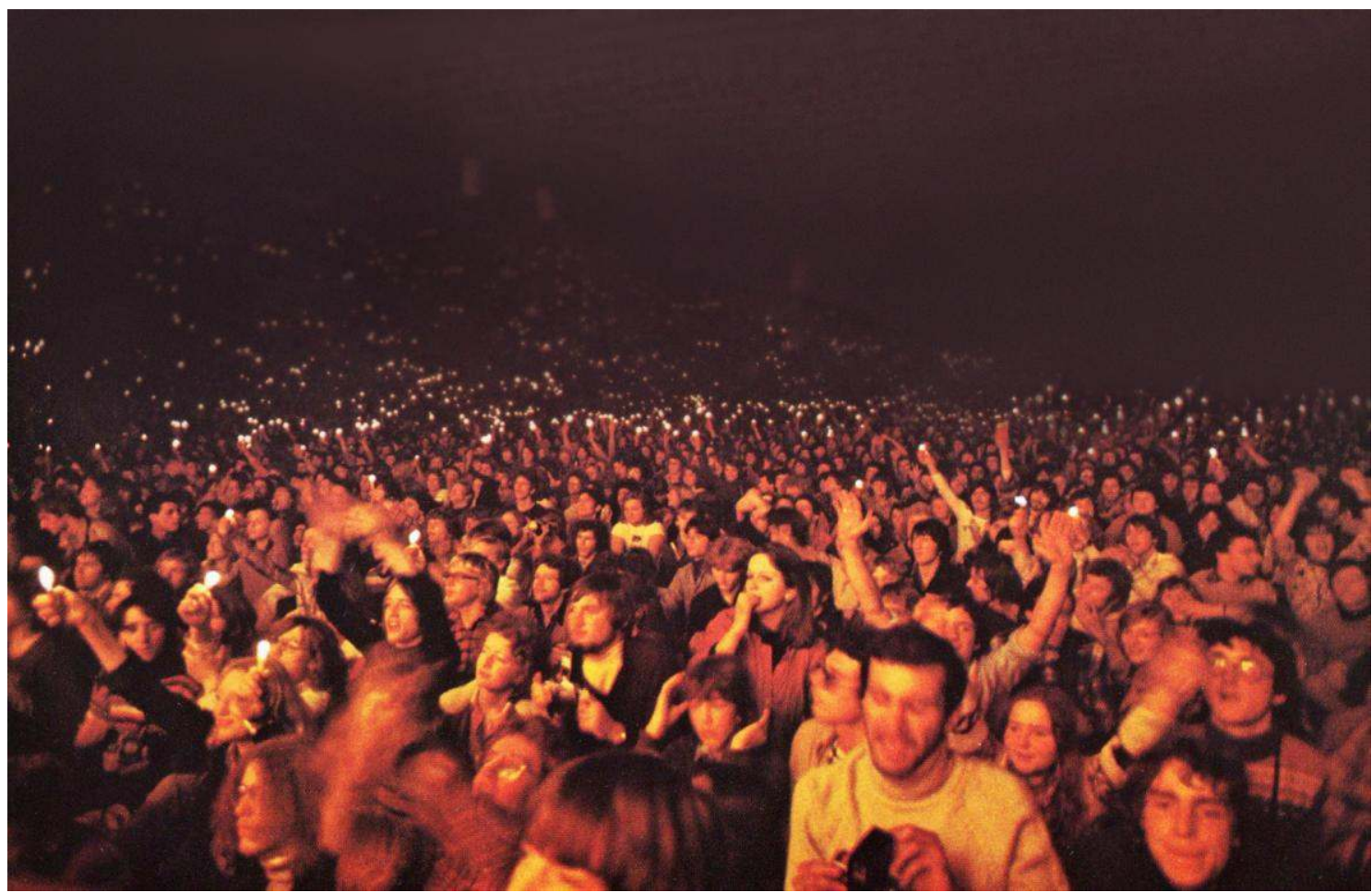

Figura 5: Tournée (1979), produzione Zard.

Generandosi dal deprecato riflusso, simili fenomeni popular restituivano un principio sociale, quello delineato da Guy Debord ne La società dello spettacolo, che si relazionava alle istanze della contestazione in modo tanto profondo quanto controverso. Il pensatore francese infatti aveva intravisto proprio nella società spettacolare delle immagini l'affermazione di una «falsa coscienza del tempo», in quanto fallimento di istanze del Sessantotto che lui stesso tuttavia aveva contribuito a formare con il suo testo pubblicato nel 1967 e divenuto un film nel 1973. Ed era la società italiana, sul finire del decennio, a rappresentare la massima espressione dei suoi timori. Infatti, nel 1979 Debord scriverà un'introduzione alla quarta edizione italiana de La società dello spettacolo in cui definisce l'Italia del riflusso «un paese che riassume le contraddizioni sociali del mondo intero» $\mathrm{e}$ «il laboratorio più moderno della controrivoluzione internazionale» ${ }^{48}$. Era in particolare l'episodio-culmine del terrorismo, il caso Moro, a essere interpretata da Debord nella sua dinamica mediatica come «un'opera mitologica a grandi macchinari scenici» e come massima prova dei timori avanzati dal sociologo dodici anni prima riguardo una società che sempre più esercita un dominio tramite il culto dell'immagine e del suo potenziale mistificatorio ${ }^{49}$. L'immaginazione e la creatività che permeavano il decennio, impersonate nella figura del negoziatore di ostaggi Steve Pieczenik, agente-psichiatra della CIA al servizio del governo

\footnotetext{
${ }^{48}$ DEBORD, Guy, La civiltà dello spettacolo, Milano, Baldini \& Castoldi, 1997, pp. 47-48.

${ }^{49}$ Ibidem, pp. 42-43.
} 
Andreotti, avevano rivestito anch'esse un ruolo durante quei cinquantacinque giorni di Notte della Repubblica nella primavera $1978^{50}$.

David Zard affermò sibillinamente che in Italia il terrorismo e le strategie della tensione erano una conseguenza dello stesso spirito che aleggiava sui concerti.

Non posso credere che ci siano le solite venti persone... che continuano a rompere le scatole... e la polizia non abbia niente per arrestarli... Questi sono protettissimi da qualcuno. Vogliamo parlare chiaro? Le Brigate rosse sono una conseguenza di quello che hanno fatto passare ai concerti, ragazzi siamo sinceri... Si annuncia il concerto dei Santana a Verona. "Due spari in aria danno il via al concerto dei Santana". Questo è sobillare, stampa di stato, stampa di governo, reazionaria, che sobilla il disordine. Ho tutta una documentazione su questo fatto: quando un giorno lascerò l'Italia - perché un giorno la lascerò - pubblicherò tutto. Eh vedrai come sarà bello...

Ma perché non la pubblichi adesso?

No, perché potrei venire rapito come Moro. Io ho tutta la documentazione, guarda per Lou Reed a Roma non mi possono venire a raccontare che e colpa di quaranta ragazzi, io so di chi è la colpa. Perché, quando era tutto calmo, la polizia e entrata con i lacrimogeni sparati da fuori verso la platea? Ma dove viviamo? La polizia deve mantenere l'ordine. La polizia a Milano coi Santana vede le casse assaltate e cosa fa? Si ritira... ${ }^{51}$

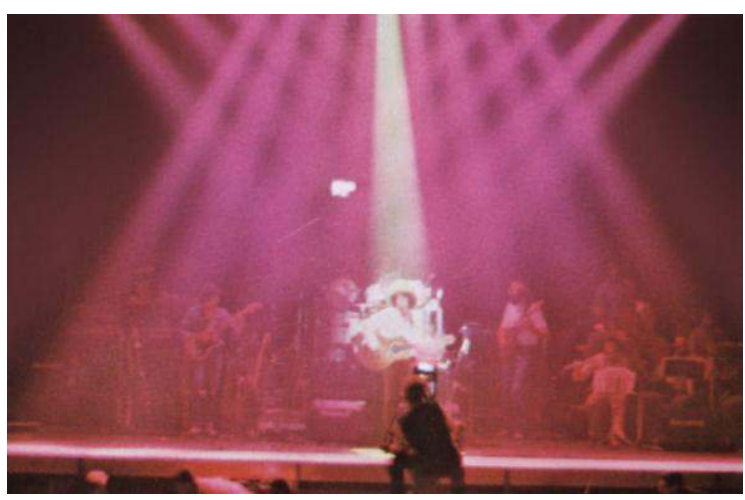

Figura 6: Tournée (1979), produzione Zard.

\footnotetext{
${ }^{50}$ Steve Pieczenik era attivo anche nel campo della letteratura e della scenografia per il cinema. Pare che si debba a lui in particolare l'ideazione del falso annuncio dell'uccisione di Moro (l'episodio del "Lago della duchessa") attribuito alle BR ma in realtà coordinato dai servizi di sicurezza dello Stato. Caso di creatività in quanto manipolazione psichica, ultimo ed estremo di un'immaginazione impadronitasi davvero dei meccanismi del potere in un modo che le più genuine contestazioni del Sessantotto mai avrebbero immaginato. «La decisione di fare uccidere Moro non fu presa alla leggera [...] Ma Cossiga mantenne ferma la rotta [...] con la sua morte impedimmo a Berlinguer di arrivare al potere e così la destabilizzazione dell'Italia e dell'Europa». AMARA, Emmanuel, Abbiamo ucciso Aldo Moro. Dopo 30 anni un protagonista esce dall'ombra, Roma, Cooper, 2015, Quarta di copertina.

${ }^{51}$ BERNIERI, Claudio, Non sparate sul cantautore, cit., pp. 141-142. L'episodio a cui si riferisce Zard è il concerto di Carlos Santana al Velodromo Vigorelli di Milano nel 1977 quando volarono perfino bombe molotov sul palco e venne esposto lo striscione Santana servo della CIA. Cfr. anche BIACCHESSI, Daniele, Storie di Rock italiano, cit, p. 105.
} 
Riguardo ai grandi spettacoli musicali popular di quel periodo, non è facile a tutt'oggi risalire alle reali cause di quelle violenze. Un fattore di contestazione da parte del pubblico risiedeva certo nell'ideale diffuso che la musica in quanto bene popolare dovesse essere accessibile a tutti, e quindi i concerti, come i dischi, costare poco o nulla. Pare tuttavia che certi slogan come «la musica è nostra e riprendiamocela» oppure «la musica si ascolta e non si paga» scaturissero anche da equivoci e sobillazioni esterne ${ }^{52}$. I musicisti e i cantanti venivano contesi anche in ragione dei contenuti, politici o meno, della loro musica, come testimoniò la lettera aperta che De Gregori pubblicò a mo' di autodifesa dopo i fatti del Palalido ${ }^{53}$, e le strumentalizzazioni erano all'ordine del giorno. Studi come quelli condotti da Marilisa Merolla o informative come quelle di Mimmo Franzinelli ${ }^{54}$, hanno fornito sì alcuni elementi, che tuttavia non riescono ancora a motivare dichiarazioni come quella di Zard che abbiamo riportato.

In ogni caso, anche a queste dinamiche, per quanto ricostruibili, vanno ricondotti i grandi spettacoli e le operazioni mediatiche in un paese che stava disattendendo i propri impegni e cercava di scacciare nelle fiabe i fantasmi di una guerra civile. Chissà che lo spirito del tempo non arridesse ad antiche storie d'altrove come le Child Ballads anche per via della violenza di quelle parole, come ricordava lo stesso Branduardi ${ }^{55}$. Dopotutto, anche Alla fiera dell'est, pur nella sua veste di filastrocca, narrava una sequela di uccisioni, incendi e bastonate. E di poteri calati dall'alto ${ }^{56}$.

\footnotetext{
${ }^{52} \mathrm{Cfr}$. Ibidem e la testimonianza di Patrizio Fariselli in Il lungo intreccio. L'onda lunga del '68 fra cultura, politica e popular music, in CARRERA, Alessandro (a cura di), La memoria delle canzoni. Popular music e identità italiana, Novi Ligure, Puntoacapo, 2016, pp. 36-67, p. 55.

${ }^{53}$ Lettera pubblicata sulla rivista «Muzak» nell'aprile 1976 cit. in DEREGIBUS, Enrico, Quello che non so, lo so cantare, cit., pp. 93-94; FABRETTI, Claudio, Francesco De Gregori, cit., pp. 92-93.

${ }^{54}$ FRANZINELLI, Mimmo, Rock e servizi segreti. Musicisti sotto tiro. Da Pete Seeger a Fabrizio De André, Torino, Bollati Boringhieri, 2010.

${ }_{55}^{55} \mathrm{Cfr}$. nota 45 .

${ }^{56}$ Un caso esplicito di attualizzazione politica dell'arcaica vicenda Chad Gadya è offerto dalla cantante ebraica Chava Alberstein. Nel 1989, ne avrebbe dato una propria versione dalle sonorità inquiete (in ALBERSTEIN, Chava, לונדון - אלברשטיין חוה, [London], CBS, 1989), accentuando, accentuando il carattere ossessivo delle ripetizioni e ampliando infine il testo come strumento di critica rappresentazione del conflitto arabo-israeliano, URL:

< https://www.youtube.com/watch?v=DHdVYy5B6JM > [consultato il 2 dicembre 2017].
} 


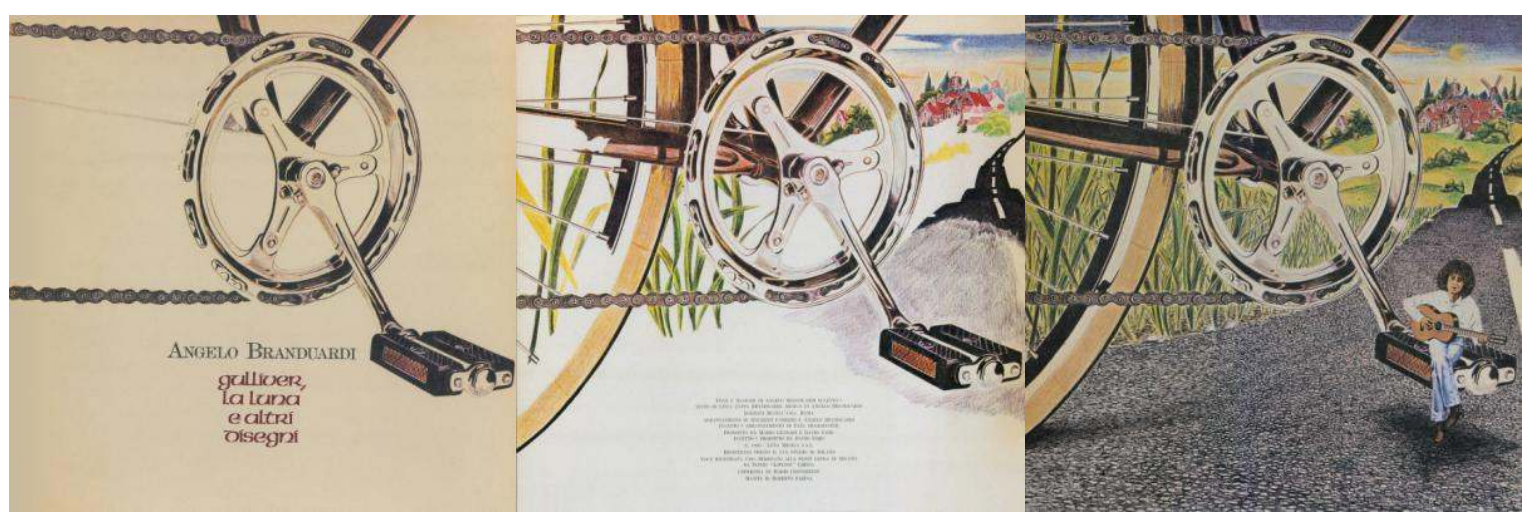

Figura 7a: copertina di Mario Convertino per Gulliver, la luna e altri disegni (1980).

Figura 7b: prima "matita" di Roberto Farina (Ibidem, interno copertina sx).

Figura 7c: seconda "matita" di Roberto Farina (Ibidem, interno copertina dx).

Al successo dell'operazione condotta da Zard concorreva una forte attenzione all'immagine anche nei prodotti discografici, in quanto oggetti estetici. I Long-Playing incisi da Branduardi per la Polydor erano ricchi album da sfogliare dall'accattivante veste grafica. Per illustrare i testi dei brani venivano inclusi scatti fotografici o addirittura, nel disco La pulce d'acqua, tavole disegnate dal noto grafico Mario Convertino, allegate separatamente in un raccoglitore interno. Le immagini erano raramente descrittive di testi e titoli, ma anche questa era una poetica "di scarto" tipica del periodo. Nel 1980 per la ri-edizione del LP La luna, col titolo Gulliver, la luna e altri disegni, la copertina di Mario Convertino (Fig. 7a) lasciava spazio a due arricchimenti creativi nelle pagine interne (le "matite" di Roberto Farina, Fig. 7b-7c). Il disco includeva persino un album di fogli bianchi per stimolare attivamente il pubblico alla creazione di commenti visivi, paesaggi, personaggi e racconti intorno a quelle canzoni pre-Zard (con l'inedito Gulliver che segna un ritorno di Paul Buckmaster ad arrangiare un antico canto bretone). Pure così si esprimeva uno spirito del decennio appena trascorso che aveva convogliato il principio della fantasia nella creazione di immagini anche in ambito musicale. Una tendenza che, più di Convertino, forse più di tutti, era ben rappresentata da Gianni Sassi e dalle sue immagini per le copertine dei dischi Cramps che rientravano in una più ampia attività grafica condotta da Sassi in altri settori artistici, culturali e commerciali.

Le caratteristiche audio-visive e la concezione dei gremiti concerti di Branduardi in quel periodo sono testimoniate non solo dal citato triplo LP Concerto, ma anche dal film omonimo realizzato nella medesima circostanza e poi distribuito nelle sale. Vengono mostrati momenti dei concerti alternati a interviste e fasi di preparazione e socialità attorno al palco: qualità filmiche trascurabili, ma la pellicola è utile appunto come documento. L'operazione Branduardi non era scatenante solo per l'audience italiana. David Zard aveva intuito le valenze artistiche e commerciali di un prodotto che poteva andare ben oltre. Venne tentata un'operazione che non 
era solo quella dell'italiano portato all'estero, ma dell'artista internazionale. Dischi tradotti e cantati in altre lingue e un riscontro inaudito per un cantautore italiano, soprattutto in Germania (anche dell'Est), con un pubblico che negli anni a seguire si sarebbe rivelato più affezionato di quello del nostro paese. Il picco del successo, emblematico nell'esibizione alla Fête de l'Humanité di Parigi l'8 settembre 1979, al cospetto di 200.000 spettatori, si prolungava tramite un'ulteriore produzione live internazionale nel 1980-1981 insieme a Stephen Stills, Graham Nash e Richie Havens (esibitisi però separatamente). Ragguardevoli in Italia le date sold out allo Stadio S. Siro di Milano.

\section{Riflusso negli anni Ottanta}

Il nuovo decennio passerà alla storia non solo come quello del disimpegno politico, ma in generale del ripiegamento sull'individualità, intesa negativamente in senso privato ed egoistico. Il movimento dei cantautori nel complesso sarebbe andato incontro a un conseguente calo di tensione emotiva, come Branduardi ebbe a definirlo, insieme a un decennio che da un punto di vista sociale egli alla fine liquiderà in modo caustico («L'unica cosa bella che hanno avuto questi anni Ottanta è che sono finiti») ${ }^{57}$. Parallelamente al riflusso sociale, le dimensioni del fenomeno Branduardi si affievoliscono, specie in Italia, al pari di una formula che inizia a logorarsi, nonostante la World music alle porte, e nonostante potesse dare ancora buoni risultati come colonna sonora - quella per State buoni se potete di Luigi Magni (1983) con moduli che richiamano Fiorenzo Carpi e Le avventure di Pinocchio di Comencini ${ }^{58}$.

Ad allontanare Branduardi dalla massa concorre paradossalmente proprio la sua personalità, incurante delle richieste di manager e discografici. Nel 1986 la Polydor pubblica Branduardi canta Yeats, disco minimale e intimista ormai sganciato dal côté etnico-arcaico e da ritmi di facile presa. Le poesie del Nobel irlandese per la letteratura, tradotte e adattate da Luisa Zappa, sono rese con un canto sussurrato e l'accompagnamento strumentale quasi esclusivo del rodato duo con Fabrizio. Le ricercatezze nell'ordito delle due chitarre sono anche timbriche, tramite sottili effetti sulle corde delle Pregiate Liuterie Milanesi ${ }^{59}$. Il disco nasceva anche dal credito che il cantautore si

\footnotetext{
${ }^{57}$ Servizio Videomusic relativo a Il ladro (Polydor 1990), cfr. nota 24.

${ }^{58}$ MAGNI, Luigi, State buoni se potete, RAI - Excelsior Cinematografica, Italia, 1983, 149'. Colonna sonora insignita del David di Donatello.

${ }^{59}$ Cfr. il servizio-intervista di CARPI, Andrea, "Angelo Branduardi. Chiudi gli occhi, suona e vedi», in Chitarre. Rivista di cultura e tecnica chitarristica, 20, 11/1987, pp. 18-22. Le tecniche di intreccio fra le due chitarre sono discusse in modo approfondito con cenni all'impiego dei capotasti e alla tecnica delle accordature aperte. Vengono pubblicate anche trascrizioni della prima poesia-canzone, I cigni di Coole, e di Confessioni di un malandrino (pp. 34-39). Entrambe le trascrizioni sono state effettuate da Giovanni Palombo sia in notazione, sia in intavolatura. L'intervista curata da Andrea Carpi informa anche su certi modelli chitarristici del duo, in particolare quello folk formato da Bert Jansch e Jan Renbourn (Ibidem, p. 20). Alcune
} 
era guadagnato presso il grande pubblico e fra discografici, e quindi da una conseguente libertà di produzione: «Dopo 10-12 anni di attività a questi livelli è evidente che posso fare un po' quello che mi pare. Mentre mi rendo conto che altri, viste le grandi difficoltà che hanno i giovani talenti incontrano nell'iniziare, non potrebbero permettersi le stesse cose. Magari quanti altri vorrebbero incidere un disco su Yeats o qualcosa del genere, ma chi glielo consentirebbe? ${ }^{60}$. Tuttavia il disco, che stando alle intenzioni dei due era destinato ad avere un seguito, nella stessa direzione poco mainstream, e che lo stesso Branduardi definì «ancora più strano" ${ }^{61}$, segna anche la fine della collaborazione con David Zard - il quale avrebbe ammesso che la posizione di Branduardi, sempre fedele alla sua linea artistica e mai disposto ad adeguarsi, era stata proprio la causa della loro separazione, perché secondo Zard invece bisognava andare incontro a quelle che sono le esigenze del pubblico.

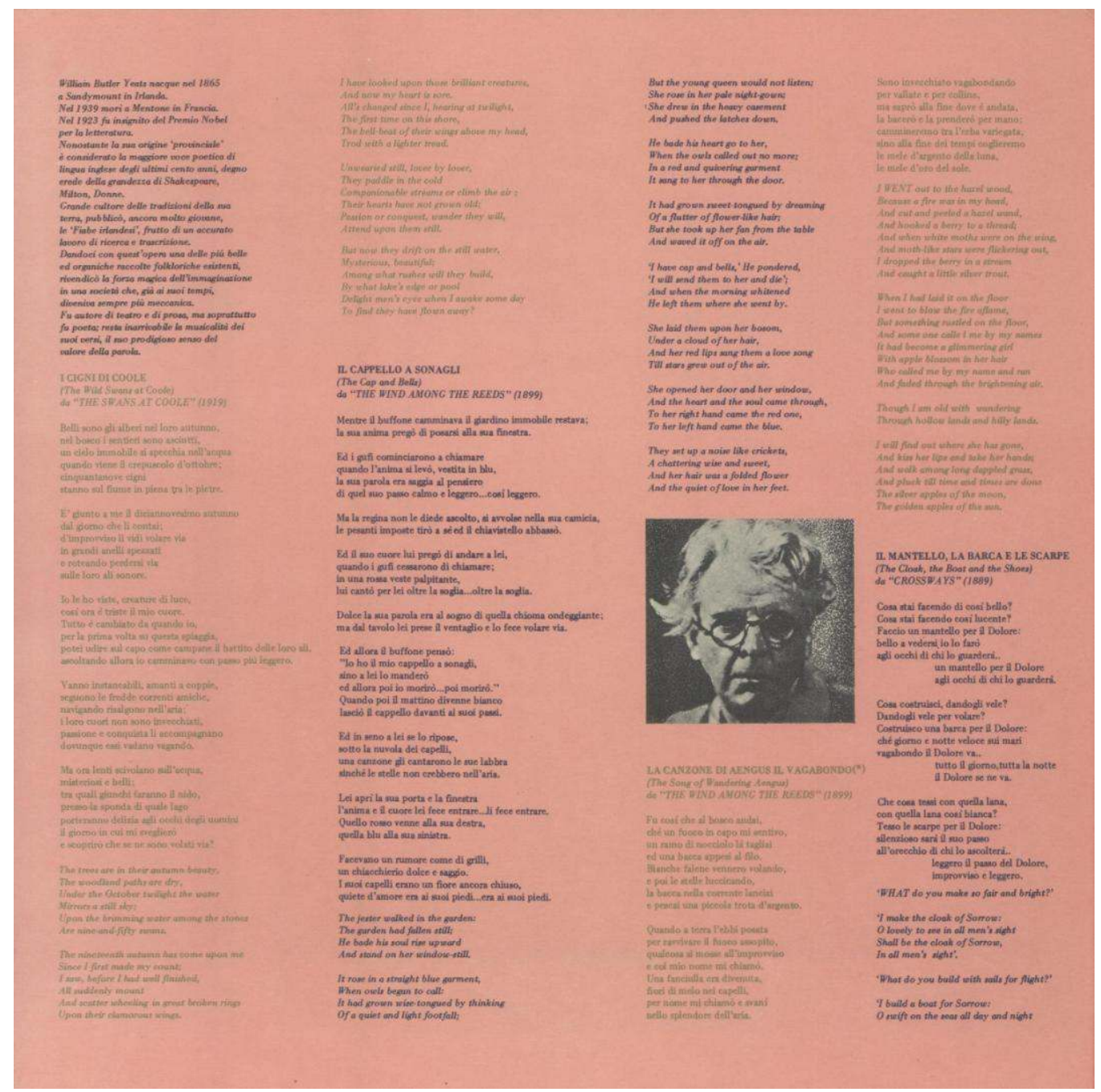

Figura 8: Branduardi canta Yeats (1986): riproduzione parziale dell'interno della copertina.

canzoni di Branduardi canta Yeats si possono ascoltare anche nel CD live Senza Spina (Sottosopra - EMI 2009) registrazione di un concerto all'Olympia di Parigi durante quella tournée del 1986.

${ }^{60}$ CARPI, Andrea, Angelo Branduardi, cit., p. 21.

${ }^{61}$ Ibidem. 
Al cospetto di questa rottura con un certo tipo di politica artistica e commerciale, l'ampio servizio curato da Andrea Carpi nel 1987 sulla rivista specialistica «Chitarre» testimoniava quanto la scelta "alternativa" di Branduardi non corrispondesse a un esclusivo ripiegamento personale, ma finisse col riscuotere i consensi di un altro circuito rispetto alla audience precedente, certo non altrettanto cospicuo in termini numerici e tuttavia fortemente connotato: quello dei musicisti o ascoltatori intenditori che al giorno d'oggi vengono definiti MUSOS (incrocio fra musicista e musicologo) secondo un neologismo coniato dal semiologo Philip Tagg ${ }^{62}$. Il fatto poi che i fraseggi chitarristici di Branduardi canta Yeats non fossero espedienti tecnici fini a sé stessi, bensì funzionali al modus poetico complessivo delle canzoni, fra parole e suoni ${ }^{63}$, convincendo gli eredi del poeta della consonanza della musica rispetto al mondo poetico del padre ${ }^{64}$, coinvolge quei circoli di studiosi e acculturati che recentemente dibattono in modo sempre più raffinato e circostanziato dei rapporti fra poesia e musica nella forma della canzone.

Il dibattito ha raggiunto un climax mediatico nel 2016 con il conferimento del Nobel per la letteratura a Bob Dylan. Il disco di Branduardi, pur additando nel breve profilo iniziale la «inarrivabile musicalità» dei versi inglesi di Yeats e il suo «prodigioso senso del valore della parola», si inserisce in questo dibattito - e nel dibattito più generale su poesia per musica / poesia in musica e musica per poesia ${ }^{65}$ - musicando traduzioni adattate per l'occasione che sovvertono le metriche assonanti degli originali riportati a fianco (Fig. 8). Peraltro, il progetto su Yeats era contemporaneo alla collaborazione con Michael Ende, il quale aveva contattato Branduardi affinché componesse la colonna sonora per un film tratto dal racconto Momo. Il film, prodotto nel 1986, non ebbe troppa fortuna ${ }^{66}$, ma l'esperienza avrebbe portato il cantautore a musicare anche alcune poesie dello scrittore tedesco ${ }^{67}$.

${ }^{62}$ TAGG, Philip, Music's Meanings. A Modern Musicology for non-Musos, New York - Huddersfield, the Mass Media Music Scholars' Press, 2013. Per muso si intende dunque un appassionato di musica con atteggiamento serio quando non elitario che spesso suona uno strumento e che parla di musica nei suoi aspetti tecnici, strutturali e creativi. Seguendo Alessandro Carrera: «il muso è quello che cammina a testa bassa ragionando di musica con altri musos come lui, e che non solo ha tutte le discografie stampate in testa della musica che ascolta, le cronologie e le biografie dei musicisti, ma sa anche distinguere gli strumenti, conosce le tecniche di registrazione, le regole dell'armonia non gli sono ignote, magari sa perfino leggere uno spartito e sa dove mettere le mani se prende in mano uno o due strumenti con i quali ha una certa pratica. Il muso è la versione metropolitana, post-borghese, arruffata e un po' barbonesca di quello che Th. W. Adorno chiamava 'l'ascoltatore strutturato'» (CARRERA, Alessandro, Introduzione, in ID., op. cit., p. 12).

${ }^{63}$ Oltre a melodie e accompagnamenti, l'allargamento dell'estensione delle chitarre tramite le accordature aperte, in particolare l'abbassamento della sesta corda da Mi a Re - quindi a partire dal basso le quinte ReLa-Re - produce un effetto-arpa, o cetra, che evoca la terra irlandese del poeta, spesso presente nei suoi versi.

${ }^{64} \mathrm{Cfr}$. l'intervista in appendice.

${ }^{65}$ Fra gli studi italiani un massimo livello di articolazione e interazione fra questi tre ambiti è delineato da LA VIA, Stefano, Poesia per musica e musica per poesia. Dai trovatori a Paolo Conte, Roma, Carocci, 2006.

${ }^{66}$ SCHAAF, Johannes Momo, Cinecittà - Iduna Film - Rialto Film, Germania-Italia, 1986, 101'.

${ }^{67} \mathrm{Cfr}$. L'intervista curata da SIMONELLI, Saverio, Michael Ende attraverso racconti di angelo Branduardi e le storie di Christian Mascheroni nell'ambito della manifestazione Michael Ende in Italia organizzata dalla "Casa di Goethe" a Roma nel 2009. URL: < https://www.youtube.com/watch?v=m2eqgjvDmjQ > [consultato il 13 
Per le oscillazioni di stile e di riscontro pubblico che abbiamo delineato finora, una delle chiavi interpretative più efficaci è quella che il sociologo francese Pierre Bourdieu fornisce tramite i concetti di habitus, di distinzione del gusto e di campo sociale. Non è possibile riassumere qui i lineamenti di un pensiero assai articolato e dalla forte di portata interdisciplinare, solo recentemente applicato anche al campo della popular music, ma del resto oggi ampiamente accessibile anche al pubblico italiano ${ }^{68}$. Basti ricordare che Bourdieu individua delle divisioni sociali, in competizione fra loro, assai più articolate rispetto a quelle che storiografie generiche e immaginari urbani hanno connotato come innovative-vs-conservatrici e dominanti-vs-dominate. Bourdieu scorge una complessa rete "campi di forze" caratterizzati da proprie dinamiche interne, di ordine economico e culturale, con le relative implicazioni simboliche e varie forme di capitali culturali, oltre che economici, da cui si origina un «mercato dei beni simbolici» al quale è associata una "produzione della credenza» che comporta contemporaneamente adesioni e opposizioni a vari livelli, interne a ogni campo, quanto fra i vari campi. In questa complessa struttura di antagonismi, i vincoli inevitabili, e perfino inconsapevoli, scorrono al fianco di margini di libertà anche individuale. Per quanto riguarda l'arte, le traiettorie del pubblico e degli artisti sono soggette tanto ai condizionamenti, quanto alle possibilità di movimento che i vari campi offrono. Un habitus che intreccia di continuo azione intenzionale soggettiva con piste regolanti oggettive conduce gli agenti artisti e fruitori a informare campi dominanti e reazionari, per certi aspetti, ma allo stesso tempo subalterni e rivoluzionari, per altri ${ }^{69}$.

novembre 2017].

${ }^{68}$ Per una panoramica cfr. PAOLUCCI, Gabriella, Introduzione a Bourdieu, Roma-Bari, Laterza, 2011; per il concetto di «campo» in generale, BOURDIEU, Pierre, Sul concetto di campo in sociologia, Roma, Armando Editore, 2010; per l'ambito letterario ID., Le regole dell'arte, Milano, Il saggiatore, 2005; per l'ambito della popular music, HESMONDHALGH, David, «Bourdieu, the Media and Cultural Production», in Media, Culture \& Society, 28, 2/2006, pp. 211-231; WILSON, Carl, Let's Talk about Love. Why Other People Have such Bad Taste, New York - London, Bloomsbury, 2014 [trad. it. Musica di merda. Parliamo d'amore e di Céline Dion, ovvero: perché pensiamo di avere gusti migliori degli altri, Milano, Isbn Edizioni, 2014].

${ }^{69}$ In una delle sintesi più chiare ed esaustive del pensiero di Bourdieu sulla funzione e ricezione dell'opera d'arte, quella scritta da Anna Boschetti per Le regole dell'arte, la fondamentale nozione di habitus «implica il superamento di una serie di false alternative che Bourdieu riconduce all'opposizione fra due modi di conoscenza, "soggettivismo" e "oggettivismo". Il mondo non è solo l'effetto delle rappresentazioni e della volontà dei soggetti, come se le disposizioni degli individui non fossero plasmate dalla loro traiettoria, né semplice manifestazione di regole oggettive iscritto nella struttura sociale, come se gli agenti con i loro punti di vista e le loro lotte non contribuissero al funzionamento della realtà. Il sociologo deve quindi ricostruire sia i rapporti di forza, sia le disposizioni e la percezione degli agenti». BOURDIEU, Pierre, Le regole dell'arte, cit., p. 17. 


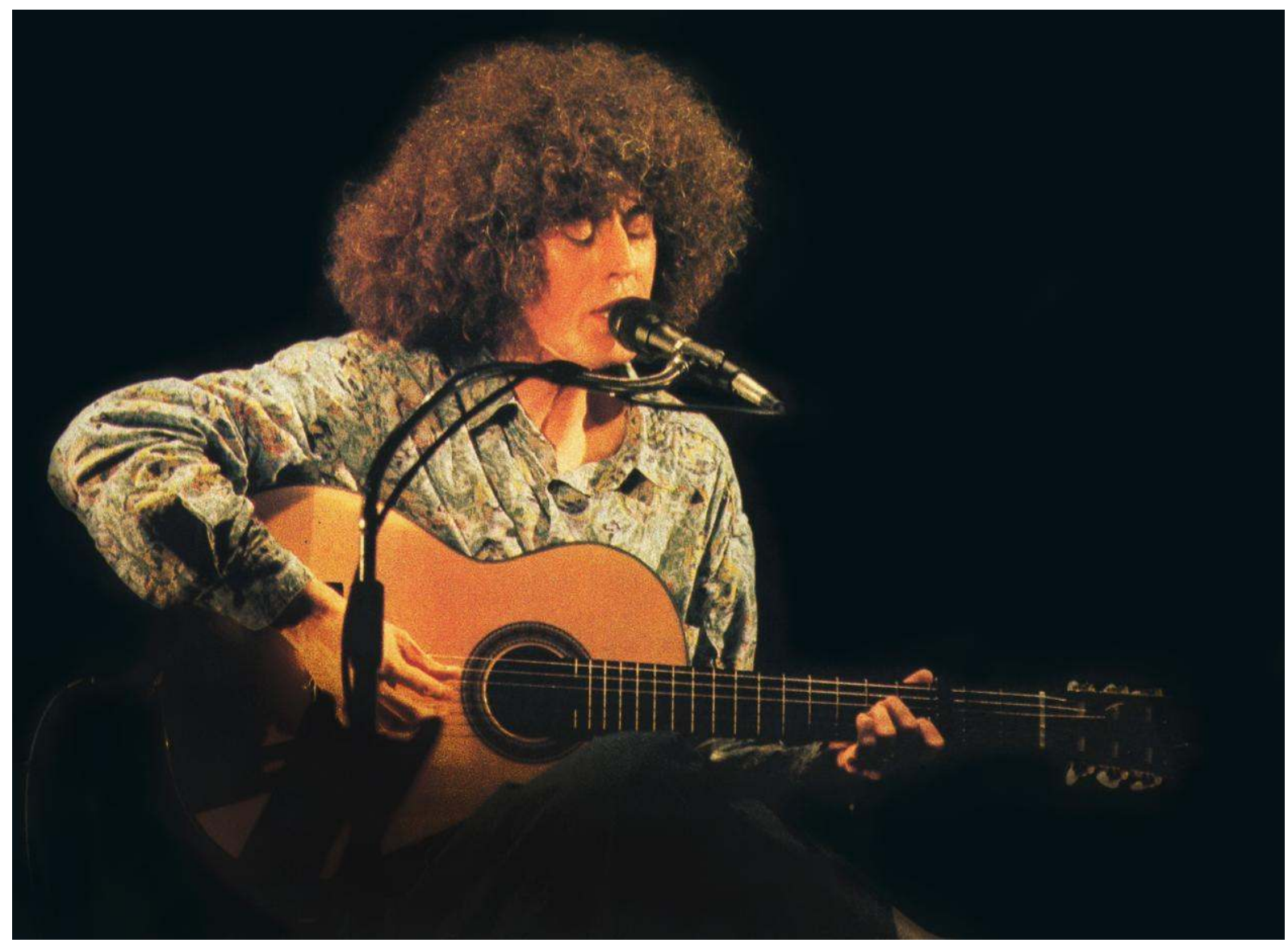

Figura 9: dalla colonna sonora per il film Momo (1986).

L'apporto più considerevole di tale teoria alla musica, popular e no, è dunque di aver evidenziato che quando si parla di scelte libere, di stili e linguaggi alternativi, o addirittura di contestazione, bisogna sempre considerare per quale campo, e rispetto a quale altro o altri campi, siffatti termini assumono significato. Come il pensiero di Guy Debord, anche quello di Bourdieu ha goduto di ampia diffusione in epoca post-Sessantotto, soprattutto per la sua critica al sistema scolastico francese e la sua analisi di certi sistemi, anche mediatici, che a suo dire esercitano il potere tramite forme di «violenza simbolica». Negli anni Settanta, proprio un meccanismo come quello che fa scaturire l'operazione Zard-Branduardi - i.e. il rifiuto di aderire alle richieste di un certo establishment produce un fenomeno opposto ma altrettanto di massa - può essere letto efficacemente come dialettica fra campi antagonisti dove i rispettivi mercati e capitali simbolici informavano istanze di innovazione e/o conservazione che in modi differenti si intrecciavano anche al loro interno.

Questo avveniva in un periodo di forte crisi del paese, caratterizzato da instabilità istituzionali e da un allargamento quasi violento della sensibilità politica, quando le scelte di ascolto da parte del pubblico, come quelle creative da parte degli artisti, corrispondevano più che mai a comportamenti sociali fortemente connotati: dove tuttavia si aprivano certi spazi di libertà (o "lacune strutturali", come le definisce Bourdieu) per strategie culturali e di immagine che 
potevano sovvertire la distribuzione esistente delle possibilità e dei profitti economici. In tal modo, il sistema sociale stabilisce un rapporto biunivoco fra le proprie possibilità e la libertà dell'artista.

Le lacune strutturali di un sistema dei possibili che probabilmente non si dà mai come tale nell'esperienza soggettiva degli agenti (contrariamente a ciò che potrebbe far credere la ricostruzione ex post) non possono essere colmate dalla virtù magica di una sorta di tendenza del sistema all'autocompletamento: il richiamo che esse racchiudono è udito solo da coloro che, per effetto della loro posizione nel campo, del loro habitus e del rapporto (spesso di discordanza) fra i due, sono abbastanza liberi nei confronti dei condizionamenti insiti nella struttura da poter cogliere come se fosse qualcosa che riguarda solo loro, una virtualità che in un certo senso esiste solo per loro. Il che conferisce alla loro impresa, a cose fatte, l'apparenza della predestinazione ${ }^{70}$.

\section{Coda}

Il dopo-Zard vede Branduardi produrre due album per la Polydor-Sottosopra, Pane e rose (1988) e Il ladro (1990), giudicati sommariamente «meno riusciti» e «dominati dall'essenzialità in un clima di tensione sospesa» ${ }^{71}$. Piuttosto, in coincidenza di un impoverimento timbrico della voce e riandando a testi che non reggono ovviamente il paragone con quelli di Yeats, l'ex menestrello modifica il proprio sforzo creativo e dei suoi collaboratori trovando punti di contatto con incipienti tendenze fusion - quel grande tentativo di commercializzazione del jazz messo negli anni Ottanta ormai alle corde. Ritornano i moduli popolari, ora però di matrice latina, partecipa perfino il fisarmonicista Richard Galliano, mentre il violino e le chitarre acustiche di Branduardi trovano nuovi interlocutori fra voci femminili e suoni campionati. La continua ricerca timbrica debitrice del lavoro sulle colonne sonore (oltre a Momo; Luci Lontane ${ }^{72}$ e Secondo Ponzio Pilato ${ }^{73}$ ) produce sonorità aggressive oppure contenute da fraseggi minimali. Sperimentazioni che avvicinano ulteriori MUSOS e critici prima avversi ${ }^{74}$, allontanando ulteriormente la massa dei primi fans, specie in Italia.

Il recupero di un pubblico più generico avverrà negli anni Novanta, dopo il passaggio alla EMI «quando rispetto al mio passato sono arrivato a fare una specie di pop, se vogliamo anche fatto

\footnotetext{
${ }^{70}$ Ibidem, p. 316

${ }^{71}$ DEREGIBUS, Enrico, RECUPERO, Attilio (a cura di), Dizionario completo della Canzone italiana. Cantanti, autori, gruppi, produttori, Milano, Giunti, 2006, p. 73.

${ }^{72}$ CHIESA, Aurelio Luci lontane, Produzioni Intersound, Italia, 1987, 90'.

${ }^{73}$ MAGNI, Luigi, Secondo Ponzio Pilato, Massfilm, Italia, 1988, 105'.

${ }^{74} \mathrm{Cfr}$. alcune recensioni citate SIMONELLI, Saverio, La musica è altrove. Cielo e terra nella musica di Angelo Branduardi, Milano, Àncora, 2012, pp. 33-34.
} 
abbastanza bene, ma molto meno originale», ammetterà lui stesso nel $2002^{75}$. Le performance dal vivo sono sempre occasione per riproporre vecchi successi, tuttavia con un'audience che nel frattempo è cambiata molto nelle dimensioni e nei comportamenti (ma anche il cantautore un tempo molto "fisico" e poco loquace sul palco cede il passo a un intrattenitore più affabile). Meno originali sono anche i recuperi medievali e rinascimentali della serie Futuro antico, ri-arrangiati in modo talvolta complesso, ma senza l'aura cantautorale dei primi anni. Forse è scaturito ripensando al filippino State buoni se potete, il progetto L'Infinitamente piccolo dedicato alla figura di S. Francesco patrocinato dall'Unione delle Famiglie Francescane d'Italia in occasione del Giubileo del 2000, che comporta per il cantautore ulteriori colpi d'ala commerciali e di popolarità: il concerto in piazza S. Giovanni a Roma costituisce uno dei più clamorosi connubi fra popular music e mondo cattolico, (tre anni dopo il Concertone in Vaticano che aveva visto la partecipazione, fra gli altri, di Bob Dylan). Qualche acquisizione artistica deriva dagli interventi di Ennio Morricone e delle Nuova Compagnia di Canto Popolare, a fianco tuttavia di materiali riciclati da vecchi LP, oltre ai consueti prestiti celtici e rinascimentali. ${ }^{76}$ Le uscite discografiche successive aggiungeranno poco o nulla. L'attività concertistica continua però incessante e si diffonde su internet una rete di fan e social forum, in aggiunta all'ovvio sito ufficiale del cantautore, che ne ravvivano la presenza mediatica caricando sul canale YouTube filmati e registrazioni utili anche per la documentazione del passato.

Figura 10: Maurizio Fabrizio in tournée con A.

Branduardi, Claudio Guidetti ed Ellade Bandini (1996).

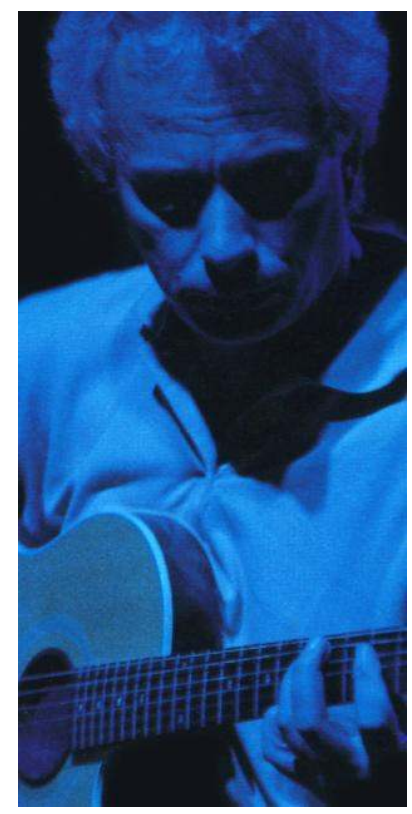

Le dichiarazioni riportate in appendice sono state raccolte il 25 gennaio 1997 al Centro sportivo di Roncadelle, piccolo paese alle porte di Brescia, dove Branduardi ha tenuto un concerto nell'ambito di un Happening organizzato dal Segretariato Oratori della città. Fra le varie iniziative, nel pomeriggio prima del concerto venne organizzato un dibattito con il cantautore. L'istituzione cattolica bresciana, coordinata allora da Don Amerigo Barbieri, aveva già da tempo intenzione di coinvolgere il cantautore. Nel 1997 era stato appena pubblicato il primo numero della serie «Futuro antico» e il concerto si inseriva in quella tournée. Durante il dibattito, trascritto solo in minima parte sulla rivista «BresciaMusica» ${ }^{77}$, si era parlato della musica di Branduardi in senso

\footnotetext{
${ }^{75}$ Intervista con BARONE, Ricky, Note alte. Sette chiavi di lettura fra Dio e musica, Brescia, Meccanica delle idee, 2014, p. 14.

${ }^{76}$ Per ulteriori considerazioni sul progetto, cfr. Ibidem.

${ }^{77}$ BIANCHI, Carlo, Il musicista non è un cantante, cit.
} 
tecnico, ma anche delle sue concezioni ed esperienze umane, sia attuali (allora) sia in senso retrospettivo. Le considerazioni sul disco Branduardi canta Yeats rievocano il lavoro insieme a Maurizio Fabrizio e chiariscono la posizione del cantautore in merito al rapporto poesia-musicacanzone - quindi al dibattito che si è sviluppato sempre di più negli anni successivi. Il disco su Yeats costituisce il punto di partenza per una disquisizione sull'atteggiamento del cantautore nei confronti dei suoi collaboratori, delle caratteristiche della sua produzione e del rapporto con il pubblico. Nel delineare una serie di percorsi e atteggiamenti, egli tende a generalizzarli ovviamente senza documentazioni e pretese di rigore sociologico. Emergono tuttavia sorprendenti analogie con quelle «regole» delineate da Bourdieu, fra strutture e margini di libertà, che durante questo saggio abbiamo ricondotto alle istanze di contestazione e riflusso nel contesto italiano degli anni Settanta-Ottanta. Il protagonista di un fenomeno popular offre la sua visione, da una prospettiva per certi aspetti privilegiata. Agli studiosi il compito dell'ermeneutica.

\section{Crediti iconografici}

Figg. 1-2-4-5-6: tratte dal triplo LP live Concerto, Polydor-Polygram 2448 112/114 (1980) variamente attribuite a Guido Harari; Jurgen and Thomas; Klaus Schlenitz ed Elvio Gallo.

Fig. 3: screen-shot dal Film Concerto, regia di L. Zappa Branduardi, prodotto da Dory Zard per la Luna Musica, Italia, 1980, 100'.

Figg. 7a-7b-7c: dal LP Gulliver, la luna e altri disegni, Polydor-Polygram 2448115 (1980) per gentile concessione EMI - Universal Music Group.

Fig. 8: dal LP Branduardi canta Yeats (1986) Polygram 829 476-1 per gentile concessione EMI - Universal Music Group.

Fig. 9: dal LP Momo. Colonna sonora originale di Angelo Branduardi, Polygram 831 296-1 (1986).

Fig. 10: dal CD live Camminando Camminando, EMI 8527502 (1996) foto di Maddalena Branduardi e Pia Neuenschwander.

L'autore si è preoccupato di rintracciare per quanto possibile gli aventi diritto, contattando sia il sito ufficiale di Angelo Branduardi, sia la EMI - Universal Music Group, e rimane a disposizione per eventuali fonti non meglio identificate. Desidero ringraziare il grafico Stefano Piovani e i dipendenti del centro copie "Planet Vigasio" di Brescia, Andrea Bontempi, Gianpaolo Medaina e Sofia Merigo, per la collaborazione alla veste delle riproduzioni. 


\section{Appendice: Intervista ad Angelo Branduardi ${ }^{78}$}

Dopo aver risposto a una domanda sulle parti scritte nelle sue canzoni, specificando che quelle delle chitarre di Confessioni di un malandrino non sono mai state scritte né prima né dopo, Branduardi ribadisce lo stesso concetto per $i$ duetti del disco su Yeats e ricorda il lavoro sulle melodie...

Angelo Branduardi: «Io e Maurizio Siamo partiti da melodie che avevo scritto nel giro di parecchi mesi. Ci ho messo quasi un anno. Quello delle melodie su Yeats era stato un lavoro duro. Ricordo che fu l'anno di quella nevicata eccezionale, a casa mia cadde un metro e quaranta di neve, ci fu tutto bloccato, le scuole... per venti giorni non potei uscire di casa, e fu sfruttando quella reclusione forzata che finii tutto. Poi però l'arrangiamento complessivo, con quell'intreccio delle due chitarre così in primo piano, io e Maurizio l'abbiamo fatto in due giorni. L'abbiamo fatto io e lui, da soli, non abbiamo dovuto discuterne con nessun altro, e nemmeno volevamo farlo. Il terzo musicista, Papete [percussionista, ndr] è arrivato dopo, quando era stato deciso quasi tutto. A qual tempo io e Maurizio già suonavamo insieme da tantissimo. Torno a dire, io e lui ci conosciamo così bene.... Siamo talmente in simbiosi che io so perfettamente cosa lui farà se io suono certe cose, so perfettamente quale basso o controcanto metterà nel sentire un mio arpeggio. Sai, è uno di quei casi rari nella vita... addirittura mia moglie una volta mi ha detto: "io non sono e non sarò sarei mai gelosa di nessun'altra donna al mondo. Qualora mai fossi gelosa di una persona al mondo, lo sarei di Maurizio"».

Carlo Bianchi: Per quel disco le parole sono state un punto di partenza. La musica è venuta dopo.

A.B.: «Ovviamente, sì. In quel caso ho "cucito" la musica sulle parole. Avevo scelto delle poesie di Yeats e la traduzione l'ha fatta mia moglie. I testi mi richiedevano la massima attenzione, anche perché per tradurli e musicarli ho dovuto chiedere il permesso dei due figli di Yeats, Ann e Michael che mantenevano la massima attenzione. Data l'importanza della figura di Yeats nella storia della letteratura poetica, non mi era consentito giustamente di togliere o aggiungere una virgola. Michael Yeats, soprattutto, stava ad analizzare ogni dettaglio, per quanto riguarda i testi ma anche riguardo alla musica. Molti altri intenzionati a musicare le poesie di suo padre si sono visti negare questo tipo di permesso. Van Morrison, ad esempio, aveva già pronto un disco con le poesie di Yeats, ma alla fine la pubblicazione gli è stata negata. Non perché Morrison avesse

\footnotetext{
${ }^{78}$ Intervista realizzata il 25 gennaio 1997 a Roncadelle (BS).
} 
modificato qualcosa dei testi, ma perché secondo Michael Yeats quella musica non era appropriata, non rispecchiava il mondo poetico di suo padre».

\section{C.B.: Come vedi l'annosa questione della canzone come luogo della poesia? Quando le parole di una canzone possono essere poesia, se non lo sono già in partenza?}

A.B.: «Per me la canzone si risolve nella musica, se non del tutto almeno per un $90 \%$. Dopodiché, però, anche le parole per me sono musica, hanno una loro musicalità. Per me tutta questa questione di lana caprina sulla poesia e la musica e i loro rapporti è abbastanza semplice. La poesia, quando è bella poesia, è già a suo modo musica e sta in piedi da sola. Altrettanto, la musica quando è bella musica sta in piedi da sola. Poi esiste una forma musicale che si chiama canzone in cui succede che le due cose convivono e siano indissolubili: il testo e la musica non stanno in piedi da sole e se si separano la canzone perde il proprio senso. Vorrei dire di più: il cromosoma della canzone, l'atomo da cui scaturisce tutto, è costituito da una nota e da una sillaba. Siccome la sillaba di per sé non vuol dire niente, è quando viene unita alla nota che riesce a "catturare". Quando i bambini cantano o dicono ABBRACADABRA, cantano una parola che in sé non vuole dire niente, però per loro riflette un significato. Ciò vuol dire che quando le parole vengono cantate $\mathrm{e}$ in generale unite alla musica, vengono caricate di un senso che va al di là dei loro significati semantici e che è anche di carattere sonoro. Nella canzone convivono significati sonori e semantici, che non sono la stessa cosa».

\section{C.B.: Il tuo percorso artistico fino ad oggi è sembrato improntato a una coerenza di fondo.}

A.B.: «In realtà non la vedo come una questione di coerenza, e da certi punti di vista nemmeno di volontà. Molto spesso gli artisti dicono di sé quello che non sanno. Per cui alla fine c'è un ordine definito, che è inconsapevole. Il pregio della musica dignitosa e sincera, quindi del fatto di non pensare a cosa uno deve fare ma di lasciare che la musicalità mostri la via è questo, cioè che lei sa dove deve andare. Basta che uno la segua. Uno nasce con questo naso qui, e nasce con quella musicalità lì. È inutile "resistere", o stare a pensare di fare altre cose... Io per quattro-cinque anni ho passato un periodo di introversione completa, in cui non comunicavo nulla, perché non avevo voglia di comunicare nulla. Era un muro. Era una cosa solo per me. Quantitativamente il pubblico è scemato, a parte uno zoccolo duro di branduardiani, a cui peraltro consiglio una visita psichiatrica [risate ndr], gli altri hanno detto: "ma questo è diventato matto". Io lo sapevo benissimo, quando facevo gli album inquietanti come Il ladro, che per una buona metà non mi avrebbero seguito. Però, se in quel momento senti di dover fare quello, devi bere l'amaro calice, poi dopo capiterà qualcosa di diverso. L'artista ha una funzione che è quella di rappresentare sé 
stesso, di rappresentare l'individualismo più evidente, ma di rappresentarlo per gli altri. Non ci sono altri compiti dell'artista che non siano questo. Anche se l'arte per l'arte è un concetto che poi si è dimostrato fasullo, la creazione resta un fatto individuale. Nel momento in cui uno lo condivide con qualcun altro, riesce a condividerlo con un numero di persone il più largo possibile, in quel momento ha raggiunto quello che si dice "successo" che è un fatto puramente casuale».

\section{C.B.: Davvero, il successo è un fatto casuale?}

A.B.: «Mi spiego meglio. Secondo me l'unica attività al mondo dove il massimo dell'egocentrismo diventa il massimo dell'altruismo è proprio l'attività artistica. Uno scrive di sé e per sé, si canta e si suona addosso seguendo un piacere, seguendo un istinto. Il dato di partenza è che il talento di un creativo è quello di avere delle antenne più sviluppate, che permettono di vedere "oltre" - che è il concetto fondamentale dell'arte: tutte quello che c'è all'interno di questa stanza all'artista non interessa: all'artista interessa immaginare cosa c'è dietro quella porta. Essendo dotato di queste antenne, di questa ipersensibilità, che in alcuni casi è anche patologica, l'artista capta delle cose che crede stiano succedendo di là, "oltre", ma un attimo prima che succedano. Se l'artista arriva nel posto giusto al momento giusto e vede questo invisibile, coloro i quali non hanno queste antenne - non perché siano inferiori bensì semplicemente diversi - dicono $\mathrm{AH}$ ! TOH! È vero! A questo punto, come dicevo prima, il "messaggio" - tra virgolette - viene decifrato e ampiamente recepito da tutti, ma in una maniera assolutamente casuale. Perché casuale? Perché se l'artista si mettesse a fare il giornalista... se si sedesse qui... se io mi sedessi qui e mi proponessi di raccontarvi una cosa precisa, mi porrei immediatamente nella condizione di prendere la mia antenna e abbassarla. Ecco cosa intendo quando dico che io "non ho volontà". Se l'artista descrive una cosa che è visibile a tutti, in quel momento il suo stesso compito decade. Egli deve essere libero, deve vedere al di là, entrare nell'altra parte dello specchio, come Alice nel paese delle meraviglie. Quando vede il paese delle meraviglie, lo racconta. Ma se tutto resta al di qua dello specchio come in una forma di volontà in cui uno si obbliga a descrivere una situazione, come farebbe un cronista... ci sono artisti che lo sanno fare bene, ma non è il mio caso. In quel caso l'artista diventa un artigiano. L'artigianato è una cosa valida quanto l'arte, ma profondamente diversa».

\section{C.B.: Che rapporto hai avuto con i tuoi collaboratori, anche quelli che avanzavano richieste?}

A.B.: «Non essendo io dotato di volontà, voi potete pure legarmi a una sedia e dirmi che devo fare una determinata cosa. Vi assicuro che non alzerò un dito. Non c'è in me alcun gusto di fare una cosa che non mi interessa. Viceversa io sono famoso nell'ambiente musicale italiano, perché quelli 
con cui lavoro - che, bontà loro, sono felici di lavorare con me - nel sentire il mio nome si mettono le mani nei capelli, perché sanno che lavorare con me significa che se una cosa mi piace io sto qui per quarantotto ore a cantare, scrivere, suonare... quelli vicino a me cadono... perché c'è in me una molla, una spinta di piacere assoluto, di potenza che non è solo quella dello spirito ma anche quella del corpo che fa sì che io vada avanti come una macchina da guerra. In questo senso il gusto dell'artista è quello infantile, ma non nel senso del bambinello scemotto, ma del bambinello con tutte le sue cariche di potenza positiva e negativa, che porta l'artista ad andare avanti e fare delle conquiste. Il punto è che magari uno non ne sa il perché, ed è giusto che non lo sappia, e il fatto rimane misterioso. Altrimenti diventerebbe, ancora una volta, un fatto di cronaca... che non è meglio o peggio: è una cosa diversa. Io credo che l'arte non abbia niente a che vedere con la cronaca. Poi altri credono il contrario. Ma se l'arte fosse cronaca, Picasso non avrebbe disegnato come ha disegnato. Avrebbe scattato delle fotografie che sarebbero state sicuramente più precise dei suoi quadri. L'arte è un'interpretazione, un simbolo di qualche cosa. Il simbolo può essere clamorosamente diverso dalla cosa, né più né meno come - altra cosa di cui nessuno è convinto - l'artista quasi mai coincide con l'uomo. Tutti se ne stupiscono, ma se l'artista scrive delle cose serene significa che è un uomo inquieto. Se scrive delle cose trasgressive significa che probabilmente è un piccolo borghese, un uomo banale. Questo è normale perché l'artista cerca delle cose, cerca... Non è per fare retorica, perché poi io sono un artista popolare... ma un grande pittore come Braque parlando dei suoi quadri diceva che ogni colpo di pennello che dava era una cicatrice in più e una ferita in meno. Ora, lui parlava da genio dell'arte, io non mi atteggerei proprio in quel modo, però il senso è sostanzialmente questo. Se uno cerca consolazione al proprio fatto, la cerca nell'esatto opposto. L'uomo disperato cerca il proprio contrario. Questo concetto va tenuto sempre presente quando si ascolta qualcosa».

\section{C.B.: È quindi in questo che trova ragione la natura delle tue canzoni? Ripeti spesso che la musica per te non è lanciare dei messaggi.}

A.B.: «Io trovo che quelli che lanciano messaggi siano persone di cui bisogna diffidare. Lo dico molto francamente. Quando sento un cantante che fa il profeta, che ci racconta parola di verità, mi viene la pelle d'oca. Gli artisti non sono dei pragmatici. Non sanno. Sapete di più voi. Non voglio esagerare, ma gli artisti nella vita civile vanno presi per mano e portati da qualche parte. Sono persone "diverse", che hanno degli equilibri "diversi", a volte oscillano da una parte all'altra. Non me lo invento io. Se leggete un qualsiasi libro di divulgazione psicologica, ve lo dirà molto chiaramente. Gli artisti hanno delle contraddizioni intrinseche, assolutamente dichiarabili, e - aihmé - non tutte positive, perché altrimenti uno non salirebbe su un palco. Questo io tendo a dirlo in modo quasi provocatorio, ma chi vi racconta il contrario mente. Gli artisti 
tendenzialmente sono egoisti, e non sempre sono gradevoli. Possono sì avere tante cose gradevoli, ma non sono persone facili. Sono persone con cui non è facile convivere, hanno degli alti e bassi, a volte violenti, sono molto spesso chiusi in sé stessi perché a sé stessi dedicano molto tempo. Io stesso non ho un carattere facile, mia moglie lo potrebbe testimoniare. Per un'artista hanno degli equilibri diversi anche le cose più piccole. Dopodiché, quelli che lanciano i messaggi ci sono eccome. Ma quando uno decide di lanciare un messaggio, questo messaggio non è mai profondo. Il messaggio è potente quando non lo si sa, che è un messaggio».

\section{C.B.: Potresti fare un esempio relativo a una tua canzone?}

A.B.: «In particolare ne ricordo uno, e lo ricordo con molta simpatia. Durante i primi concerti che facevo, quando avevo appena inciso Alla fiera dell'est, notavo che in sala c'erano spesso molti religiosi, e mi chiedevo come mai. Una sera, all'Aquila, venne da me una suorina molto carina, la quale mi dice "Lo sa, signor Branduardi, che mi piacciono molto le sue canzoni, soprattutto quella che parla della resurrezione". Io rispondo: "la ringrazio, ma guardi che lei ha sbagliato cantante, deve aver sentito un altro concerto. Io non ho mai scritto canzoni sulla resurrezione". "Certo che sì" ribatte lei. "E quale sarebbe?" domando. "Il dono del cervo..." Io sono rimasto un po' a pensare, interdetto. In realtà avevo scritto questa canzone perché mi piaceva l'idea della fiaba, del cavaliere e del cervo che dona le sue corna, la sua pelle, i suoi occhi... ma in quel momento, di fronte alla suorina, mi resi conto che quella della mia intenzione originale poteva essere una lettura superficiale, e infine le dissi: "Sa, ripensandoci, forse lei ha ragione..." Mi resi conto, insomma, che l'efficacia di un significato può andare al di là della coscienza del suo autore».

\section{C.B.: Eppure nel tuo disco Domenica e lunedi c'è una canzone, Tenera nemica, che sembra abbastanza esplicitamente dedicata a una delle tue figlie e alla conflittualità di un rapporto. L'argomento ci interessa perché noi che operiamo nell'ambito degli oratori abbiamo spesso a che fare con gli adolescenti.}

A.B.: «Quando ho scritto quella canzone mia figlia aveva diciotto anni. Era il periodo, sacrosanto, in cui l'adolescente per trovare la propria identità deve discuterne con violenza un'altra. Le autorità che mette in discussione sono ovviamente due: la scuola e i genitori. È ovvio. Se io non posso fare dei tentativi e non ho uno specchio che mi restituisce un'immagine, la mia diversità non la troverò mai. Ecco perché parlo di "tenera nemica". Questa tendenza alla contestazione è normale, e per fortuna che esiste, altrimenti saremmo tutti la clonazione di nostro padre o di nostra madre. E vivremmo in un mondo assolutamente mefitico». 


\section{L'AUTORE}

Carlo BIANCHI si è addottorato nella Facoltà di Musicologia dell'Università di Pavia-Cremona dove ha ricoperto incarichi di insegnamento e ricerca. Ha pubblicato oltre 200 scritti. I suoi contributi alla sociologia della popular music sono comparsi sia su periodici divulgativi («BresciaMusica», «Critica minore») sia accademici di riferimento nazionale («Musica/Realtà», «Nuova Rivista Musicale Italiana», «DBI-Treccani») e internazionale («Iaspm-journal», «Vox popular»). Una sua lettura musicale dell'Italia anni Settanta è offerta dal saggio: Il lungo intreccio in CARRERA, Alessandro (a cura di), La memoria delle canzoni. Popular music e identità italiana, Novi Ligure, Puntoacapo editrice, 2016, pp. 36-67.

URL: < http://www.studistorici.com/progett/autori/\#Bianchi > 